\title{
WestVirginiaUniversity
}

THE RESEARCH REPOSITORY @ WVU

Graduate Theses, Dissertations, and Problem Reports

2011

\section{Outage Probability in Multimodal Networks}

Ricky E. Hussmann

West Virginia University

Follow this and additional works at: https://researchrepository.wvu.edu/etd

\section{Recommended Citation}

Hussmann, Ricky E., "Outage Probability in Multimodal Networks" (2011). Graduate Theses, Dissertations, and Problem Reports. 3281.

https://researchrepository.wvu.edu/etd/3281

This Thesis is protected by copyright and/or related rights. It has been brought to you by the The Research Repository @ WVU with permission from the rights-holder(s). You are free to use this Thesis in any way that is permitted by the copyright and related rights legislation that applies to your use. For other uses you must obtain permission from the rights-holder(s) directly, unless additional rights are indicated by a Creative Commons license in the record and/ or on the work itself. This Thesis has been accepted for inclusion in WVU Graduate Theses, Dissertations, and Problem Reports collection by an authorized administrator of The Research Repository @ WVU. For more information, please contact researchrepository@mail.wvu.edu. 


\title{
Outage Probability in Multimodal Networks
}

\author{
by \\ Ricky E. Hussmann \\ Thesis submitted to the \\ College of Engineering and Mineral Resources \\ at West Virginia University \\ in partial fulfillment of the requirements \\ for the degree of \\ Master of Science \\ in \\ Electrical Engineering \\ Daryl Reynolds, Ph.D., Chair \\ Matthew C.Valenti, Ph.D. \\ Powsiri Klinkhachorn, Ph.D.
}

Lane Department of Computer Science and Electrical Engineering

Morgantown, West Virginia

2011

Keywords: Multimodal Networks, Cooperative Communications, Network Information Theory

Copyright 2011 Ricky E. Hussmann 


\author{
Abstract \\ Outage Probability in Multimodal Networks \\ by \\ Ricky E. Hussmann
}

In the mid 1990's wireless researchers discovered that additional antennas located at the transmitter, receiver or both could help combat the unpredictable nature of the wireless channel. This field of research, known as MIMO (Multiple-Input Multiple-Output), became very active and has been thoroughly studied. Manufacturers have sought to incorporate these performance gains into their devices by including multiple transmit and receive antennas. However, as wireless devices such as mobile phones become smaller it becomes impractical to design a handset with multiple antennas.

Cooperative diversity is a technique that may be employed when device sizes are too small to incorporate a local antenna array. Using cooperative diversity, multiple wireless nodes cooperate to pass a message from a source to a destination. This leads to a virtual antenna array, allowing single-antenna devices to enjoy the benefits of a MIMO system. While cooperative diversity offers benefits, it motivates additional wireless nodes in the network. Even without cooperative diversity, our lives are increasingly dependent on a growing number of wireless devices. It's obvious that the density of wireless devices in daily use will increase, and that rise in popularity demands the most optimal use of node resources.

Unfortunately, it has been shown that as the density of wireless devices for a given area increases, in the limit, the capacity of the network goes to zero. Even with advances such as MIMO and cooperative diversity, it's obvious that a wireless-only future is impossible. For non-diminishing throughput, as wireless networks continue to grow in size, networks of the future will continue to incorporate additional modes of communication; wired, infrared, ultrasonic or other. In our work, we provide a strategy for harnessing these additional modes and optimizing across all the available modes of communication.

In this thesis, we present a protocol for wireless relay networks with an additional nonfading mode of communication available. As an example, we assume the presence of an additional wired channel in a relay network operating under the Laneman protocol, and we find analytical expressions for outage probability assuming communications over the wired and wireless channels are jointly optimized. We consider two cases: adding a channel between the source and a relay, and adding a channel between a relay and the destination. We show that a channel placed between the source and a relay separated by a poor wireless channel improves performance with few assumptions on the characteristics of the wire and over a wide range of wire channel transmit powers. 


\section{Acknowledgments}

I'd like to begin by thanking my advisor, Dr. Daryl Reynolds. Had it not been for his willingness to take me on a researcher, I would most certainly have not been able to complete my masters study at WVU. However, immensely more important was his patience and knowledge in dealing with a struggling researcher. It was only through his support that I have developed the tools to complete this work, and hopefully move forward in my research.

I cannot continue without thanking Dr. Matthew Valenti, not only for agreeing to participate on committee, but also for inspiring me to pursue research and an advanced degree. It was through his teaching and conversation, and a research position that was generously extended during my undergraduate career, that I decided to pursue a future in communications study.

I must also recognize Dr. Powsiri Klinkachorn. Since initially meeting Dr. Klink, he has been an advocate and mentor. He has continually showed confidence in my success, despite the many opportunities I've given him to change his opinion. I'm very lucky to have met someone who encourages me towards success, and has such faith in my abilities. For that, I'm forever grateful.

A special thank you to Dr. Natalia Schmid for helping me develop the tools necessary for my research, and for taking time from her own schedule to assist in tackling several problems along the way. Thanks also to Dr. Arun Ross; never in a bad mood, truly interested in students' lives, and refuses to pass you in the hallway without saying hello.

Many thanks to Adam Horne and John Wyatt. Not only for being fantastic friends, but also for reviewing this thesis and providing valuable feedback. In addition, I'm appreciative of all my friends for the support they've provided throughout my graduate career thus far. I must acknowledge my colleagues in the Wireless Communication Research Lab. These students are bright, friendly, and never too busy to listen. Thanks Terry, Omar, Xinghyu, Chandana, and my parter in crime, Scott. 
Finally, I must thank my family. To Waneta and Rick Hussmann, neither of whom believe I can fail (and both are certainly very wrong), I will never be able to repay you for being the best parents a son could ask for. To my sisters Lisa and Cathy, while they may prod and poke fun, I know I can count on them for anything, that's what siblings are for. To everyone in between, and my cat Nibble, the space here is not sufficient to express my thanks. So I'll end in simplicity. Thank you. 


\section{Contents}

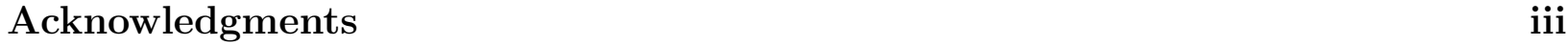

List of Figures $\quad$ vii

Notation ix

\begin{tabular}{llr}
\hline & Introduction & 1
\end{tabular}

1.1 Thesis Outline . . . . . . . . . . . . . . . . . . . . . . 3

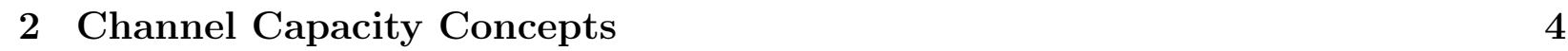

2.1 Noisy Channels . . . . . . . . . . . . . . . . . . . . . . . . . 4

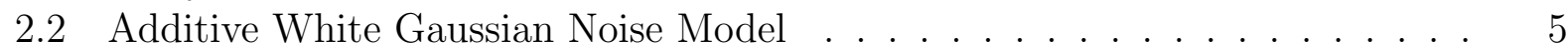

2.3 Signal to Noise Ratio and Channel Characteristics . . . . . . . . . . . . . . . 7

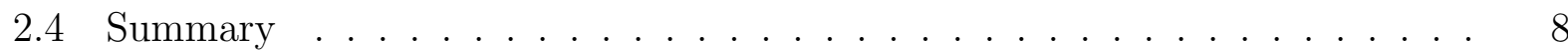

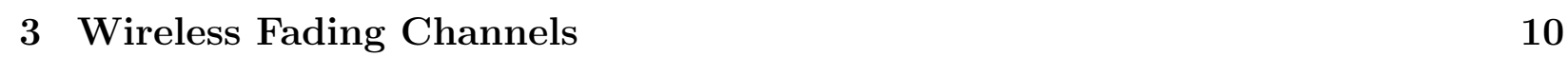

3.1 Introduction to the Wireless Channel . . . . . . . . . . . . . . . . . . . . . . 10

3.1.1 Fading Components . . . . . . . . . . . . . . . . . . . . . 11

3.1.2 Fading and the Gaussian Model . . . . . . . . . . . . . . . . . . . . . 12

3.1 .3 Channel Statistics . . . . . . . . . . . . . . . . . . . . . . . . . . 12

3.2 The Rayleigh Fading Model . . . . . . . . . . . . . . . . . . . . . . . . . . . . . . . . . . . . . . . . . . . . . . .

3.3 Diversity . . . . . . . . . . . . . . . . . . . . . . . . . . . . 16

3.4 Summary $\ldots \ldots \ldots \ldots \ldots \ldots$. . . . . . . . . . . . . . . . . . . . . 18

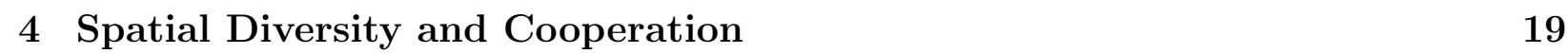

$4.1 \quad$ Overview . . . . . . . . . . . . . . . . . . . . . . . . . . . . 19

$4.1 .1 \quad$ Multiplexing . . . . . . . . . . . . . . . . . . . . . . . 19

4.2 Spatial Diversity $\ldots \ldots \ldots \ldots$. . . . . . . . . . . . . . . . . . . . . . . . 20

4.2 .1 Relaying . . . . . . . . . . . . . . . . . . . . . . . . . 20

$4.2 .2 \quad \mathrm{MIMO} \ldots \ldots \ldots \ldots \ldots \ldots \ldots$

4.2 .3 Cooperative Diversity $\ldots \ldots \ldots \ldots$. . . . . . . . . . . . . . 21

5 Multimodal Cooperation Using Repetition 24

5.1 Network Structure . . . . . . . . . . . . . . . . . . . . . . . . . . . . 24

5.2 Medium Access Control . . . . . . . . . . . . . . . . . . . . . . . . . . . . . 25

5.2 .1 Degrees of Freedom . . . . . . . . . . . . . . . . . . . . 25 
$5.2 .2 \quad$ Noncooperative Transmission . . . . . . . . . . . . . . . . . . 26

5.2 .3 Repetition Cooperation . . . . . . . . . . . . . . . . . . . . 26

5.3 System Model . . . . . . . . . . . . . . . . . . . . . . . . 27

5.4 Outage Probability Without Additional Modes . . . . . . . . . . . . . . . . . 30

5.5 Adding a Non-Fading Mode $\ldots \ldots \ldots \ldots$. . . . . . . . . . . . . . . 32

5.6 Outage Approximation for an Additional Source-Relay Non-Fading Mode . . 33

5.7 Non-fading Mode from Relay-to-Destination . . . . . . . . . . . . . . . 36

5.8 Summary . . . . . . . . . . . . . . . . . . . . . 36

6 Multimodal Cooperation Using Distributed Space-Time Coding 39

6.1 Medium Access Control . . . . . . . . . . . . . . . . . . . . . . . . 39

6.2 System Model . . . . . . . . . . . . . . . . . . . . . . . . . . . . . 40

6.3 Outage Probability Without Additional Modes . . . . . . . . . . . . . . . . . 41

6.4 Outage Approximation for an Additional Source-Relay Non-Fading Mode . . 41

6.5 Outage Approximation for an Additional Relay-Destination Non-Fading Mode 44

6.6 A Note on Path Loss . . . . . . . . . . . . . . . . . . . . . . . . . . . . . . . . . . . . . . . . . . . . . .

6.7 Summary . . . . . . . . . . . . . . . . . . . . . . . . . . . 48

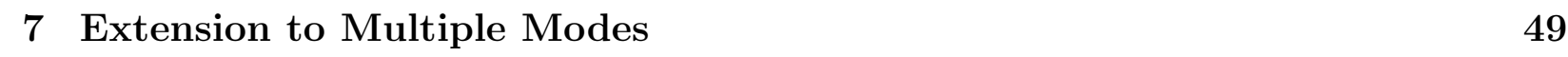

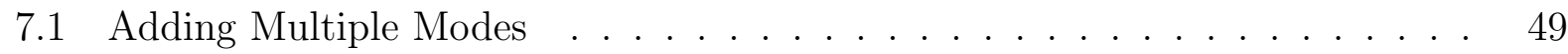

7.2 Multiple Source-Relay Modes for Repetition Scheme . . . . . . . . . . . . . . 50

7.3 Multiple Source-Relay Modes for Space-Time Scheme . . . . . . . . . . . . . 52

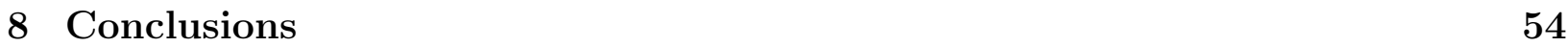

8.1 Summary and Conclusions . . . . . . . . . . . . . . . . . . 54

8.2 Future Work . . . . . . . . . . . . . . . . . . . . . . . . . . . 55

\begin{tabular}{ll}
\hline References & 57
\end{tabular}

A Additional Source-Relay Non-Fading Mode Approximation for Repetition . . 59

B Additional Source-Relay Non-Fading Mode for Space-Time . . . . . . . . . . 63

C Additional Relay-Destination Non-Fading Mode for Space-Time . . . . . . . 67 


\section{List of Figures}

$2.1 \quad$ A sample message set, and examples of received messages with noise . . . . . 6

$3.1 \quad$ Messages distorted by random rotation . . . . . . . . . . . . . . . . . . . . 13

3.2 Messages distorted by fading and additive noise . . . . . . . . . . . . . . . 13

3.3 Rayleigh PDF with parameter $\lambda=5 \ldots \ldots \ldots$

4.1 A relay network consisting of a source, $\mathrm{s}$, a relay, $\mathrm{r}$, and a destination $\mathrm{d}(\mathrm{s})$. . $\quad 20$

4.2 A two-transmitter network. $s_{1}$ and $s_{2}$ are two transmitters attempting communication with $d(s) . \ldots \ldots \ldots \ldots \ldots . \ldots \ldots . \ldots \ldots$

5.1 The time-bandwidth product degrees-of-freedom . . . . . . . . . . . . . . . 25

5.2 Medium access control for noncooperative communication. . . . . . . . . . . . 26

5.3 Medium access control for cooperative repetition communication . . . . . . . 27

$5.4 \quad$ A cooperative network with source $s$ transmitting messages to destination $d(s)$ with $m-1$ relays available for cooperation. . . . . . . . . . . . 28

5.5 Repetition outage probability for a wireless network for several values of $m$, $R=1$, and $\lambda=1$ for all channels. . . . . . . . . . . . . . . . 31

5.6 Approximation versus simulation results for a repetition scheme with $\mathrm{m}=3$, $\lambda_{s, r_{w}}=10$ all other $\lambda=1$ and $R=1$ for 12,18 and $23 \mathrm{~dB} \ldots \ldots . . . . .34$

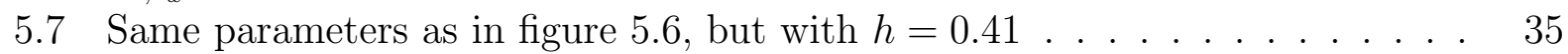

5.8 Simulated outage for a two-relay network with a non-fading mode between $r_{w}$ and $d(s)$. Figure (a) represents a network with $\lambda_{r_{w}, d(s)}=1$, (b) $\mid$ are results for

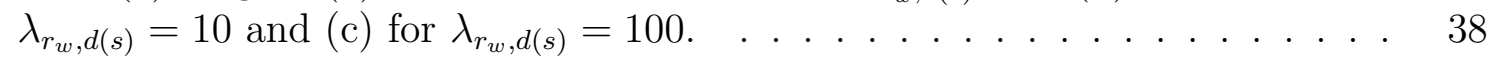

6.1 Medium access control for space-time cooperation . . . . . . . . . . . . . . . 40

6.2 Space-time outage probability for a wireless network for several values of $m$, $R=1$, and $\lambda=1$ for all channels. . . . . . . . . . . . . . . . 42

6.3 Approximation versus simulation results for a space-time scheme with an additional non-fading mode between $s$ and $r_{w}, m=3, \lambda_{s, r_{w}}=10$ all other $\lambda=1$ and $R=1$ for 12,18 and $23 \mathrm{~dB}$ when $P_{w}=0$. . . . . . . . . . . . 43

6.4 Comparison of networks with no additional fading modes versus a single nonfading mode between source and relay. Plots are for $m=3$ (blue), $m=5$ (red) and $m=9$ (green). $\lambda=1$ for all channels. $P_{w}$ is configured to allow the non-fading mode to achieve target rate $R=1 . \ldots \ldots$. . . . . . . 45 
6.5 Approximation versus simulation results for a space-time scheme with an additional non-fading mode between $r_{w}$ and $d(s), m=3, \lambda_{r_{w}, d(s)}=10$ all other

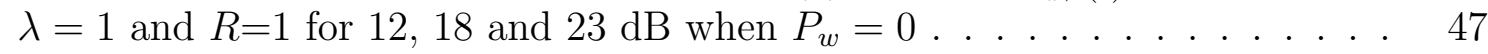

7.1 Approximation versus simulation results for a space-time scheme for a tworelay network with non-fading modes to both relays. $\lambda_{s, r}=10$ for both relays.

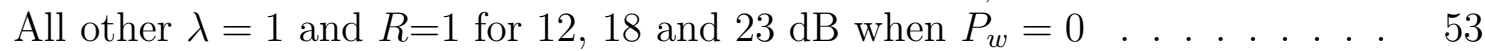




\section{Notation}

We use the following notation and symbols throughout this thesis.

$|\cdot|$ : Cardinality of a set

Calligraphic upper-case characters denote a set. 


\section{Chapter 1}

\section{Introduction}

Over the past decade, most wireless communication systems have been single-input singleoutput (SISO) systems, meaning both the transmitter and receiver possess a single antenna. While at first a single-antenna system seems intuitive, this design fails to exploit additional dimensions available for communication that could significantly improve system performance. In their groundbreaking work, Foschini and Gans [1] began to illustrate the performance gains available when multiple antennas are available at either the transmitter or receiver. Multipleinput multiple-output (MIMO) systems are wireless systems offering multiple antennas at both transmitter and receiver, and allow the system to take advantage of the random and uncorrelated nature of the wireless channel. Due to such dramatic performance gains, consumer wireless devices are integrating MIMO technology, and MIMO has been adopted into many wireless standards.

Unfortunately, for MIMO to be effective, antennas must be spaced at a certain minimum distance. For small devices such as mobile phones and other mobile units operating at high frequencies, additional antennas, not to mention minimum spacing between them, is impractical. Due to their characteristics these devices are unable to exploit the significant performance gains multiple antennas could offer. However, it is often the case that many mobile units are deployed within communication range of each other. For instance, several separate cell phones may be able to overhear a transmitting cell phone's message. These could potentially assist by transmitting the overheard information to the base station. This technique is know as cooperative diversty; wireless nodes that overhear a transmission can 
cooperate to relay that message to the destination. As a result of the cooperation, the transmitter has a virtual antenna array of multiple antennas available for transmission. While not directly connected to the transmitter, cooperating units can provide some of the benefits a MIMO system enjoys.

Cooperation motivates the existence of many deployed nodes. Ignoring cooperative techniques, it is common to find wireless technologies everyday. In fact, $93 \%$ of the US population have cell phones [2]. Sensor networks, containing hundreds or even thousands of wireless nodes are often deployed for surveillance and industrial or environmental monitoring. It's very difficult to purchase a laptop without wireless LAN technology. As research advances, it's clear the world wants to be wireless. While the demand is good news for researchers in the area, it comes at a price. Recent studies have shown that as wireless nodes become more dense, in the limit, the ability to communicate disappears. This was first shown in a geometrical framework, under a non-interference model [3]. However, additional research has shown this limitation is an unescapable physical constraint of the wireless medium [4]. Regrettably, as the demand for wireless communication grows and devices become more common, we will never see an entirely wireless world.

However, all hope is not lost. While the traditional wireless channel may be convenient, it is not the only medium available for communication. Most networks still have some nonfading mode of communication available, for instance wired communication (e.g. twisted pair or coaxial cable). Additionally, other non-wired communication modes such as ultrasonic or infrared do not suffer the same weaknesses as the wireless fading channel. In cellular networks, handsets are wireless devices, but base stations have both a wireless and wired mode of communication available. While these multimodal networks may already exist, the wireless fading mode and the non-fading modes are typically optimized separately, then joined. With a purely wireless future out of the picture, and an increasingly large number of wireless nodes competing for spectrum, future network performance gains will be obtained by considering all modes of communication available simultaneously. 


\subsection{Thesis Outline}

Chapter 1, which you're currently reading, has hopefully provided an introduction to the previous work that has led us to this point. Also, we hope that it has provided significant motivation and gravity for this work. The rest of this section will provide an outline for the remainder of the thesis.

Chapter 2 introduces the reader to a simple model for communication systems, the additive white Gaussian noise, or AWGN, model. Examples of communicating through an AWGN channel will be presented, and the concept of channel capacity will be presented. Chapter 3 establishes the Rayleigh fading channel, an extension of the AWGN model that's more representative of a wireless communication medium. This chapter focuses on the challenges present in this extended model, and provides several examples to highlight the harsh conditions. Specific assumptions for the fading channel are presented as they are important when developing a system model.

Chapter 4 approaches the topic of spatial diversity, an important weapon in combating the difficult environment of the fading channel. The chapter continues on to a specific type of spatial diversity; cooperative diversity. Motivation and benefits of cooperation are presented.

With a foundation in place, chapters 5 and 6 present the contribution of the thesis. Chapter 5 presents an existing protocol for cooperation using repetition coding. An additional non-fading mode is introduced at certain points in the network and the performance impact is analyzed. Chapter 6 presents another existing cooperative network, however a distributed space-time scheme is used rather than a repetition scheme. Once again, the performance impact of an additional non-fading mode is presented and analyzed.

Chapter 7 considers an extension of the work in chapters 5 and 6 to considering multiple additional non-fading modes of communication. Chapter 8 concludes the thesis and presents our final thoughts, as well as suggestions for future work. The last portion of this thesis is an appendix containing proofs for the various propositions presented throughout. 


\section{Chapter 2}

\section{Channel Capacity Concepts}

\subsection{Noisy Channels}

The purpose of a communication system is to convey a message across a medium. There are many mediums across which communication may occur; copper wires, coaxial and fiberoptic cable, water and air are all examples of communication mediums. There are also numerous examples of communication systems. Telephone networks, infrared remotes, broadcast radio and wired and wireless LANs are examples. During the course of a phone call, your voice (the message) may travel across multiple of these mediums on its trip to the destination (the person you're speaking with).

If it were possible to receive a message exactly as it was sent across the medium, many decades of research, and this thesis, would be unnecessary. As is often the case, though, no communication medium is ideal. Communication mediums and the equipment used for communication often distort the transmitted message. In fact, you have mostly likely experienced this phenomenon yourself. If you've ever had difficulty communicating with a friend during a football or basketball game, or had to yell at someone from a distance, you have first-hand knowledge of the degenerative effects of communication mediums. Unfortunately, these degenerative effects are present in some form in all communication mediums, and techniques must be developed to deal with noisy communication channels.

In a digital communication system, the message to be transmitted is of the form of binary digits, or bits. Often the received message includes errors. In the case of a digital 
communication system this can be thought of as "flipping bits," or randomly introducing errors in the received message. In the presence of such a hostile environment, one may initially assume it impossible to reliably communicate any information. However, in his seminal work on communication, Shannon showed that not only is it possible to achieve reliable communication over a noisy channel, he also quantified the maximum achievable data rate for error-free communication for a general class of noisy channels, the additive white Gaussian noise channel [5].

\subsection{Additive White Gaussian Noise Model}

An additive white Gaussian noise (AWGN) channel is commonly used for modeling communication systems. In this model a random perturbation is added to the message as it travels through the communication medium. Before exploring what this means quantitatively, we'll talk briefly about the validity of this model.

In most communication systems, electronic equipment is used to perform both transmission and reception of messages. This applies to both digital systems using microcontrollers and analog systems using amplifiers and reactive elements. As the electric currents flows through these components it experiences random fluctuations due to "thermal noise" [6]. These random fluctuations are a function of the temperature, and are always present. Other variations in the signal can be attributed to black-body radiation and cosmic rays from the sun. All of these factors, and numerous others not listed here, occur simultaneously and continuously, adding uncertainty to the actual value of current flow (or voltage). Ultimately, if messages are transmitted electrically at any point, they will be distorted.

To characterize the noise, we model it mathematically. As there are many sources of perturbation, we appeal to the central limit theorem to model the ensemble effect as a Gaussian random variable. Hence, we can view the output at the receiver as a combination of both the transmitted signal and a random sample of noise. Denoting the source message $x$, the output of the receiver can be written

$$
y=x+z,
$$


where $\mathrm{z}$ is a Gaussian random variable with zero mean and variance $\sigma^{2}$.

Transmitting one known message would be useless, for at the receiver we would always know the output. We would like to transmit one of a set of possible messages. Given a set of source messages $X=\left\{x_{1}, x_{2}, \ldots, x_{n}\right\}$, we would like to transmit one of the messages in the set, $x_{i}$ such that $x_{i} \in X$. Messages are arbitrary, and can be anything that we can attach mathematical understanding to. For instance, say we wish to represent messages as Cartesian coordinates. Let us create a set of two messages. Message A will be the cartesian coordinate $(-1,0)$ and Message $\mathrm{B}$ will be the coordinate $(1,0)$. Our message set is $X=\{(-1,0),(1,0)\}$. Figure 2.1(a) shows the two example messages. Figure 2.1(b) shows fifty samples of each

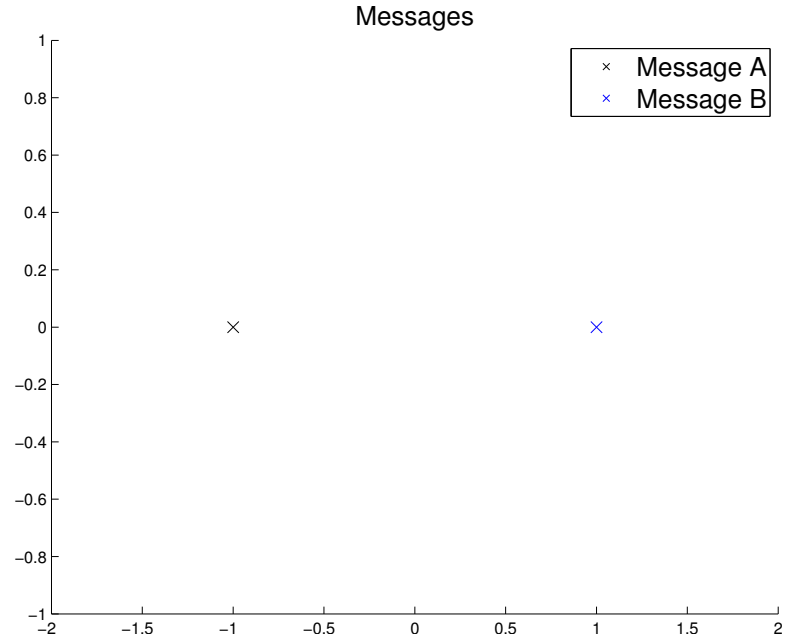

(a) Examples messages

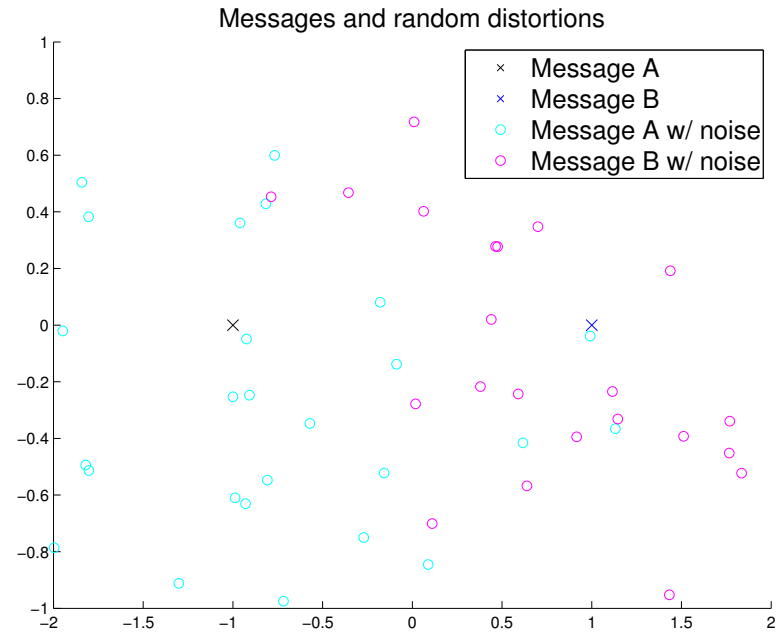

(b) Example messages shown with random noise

Figure 2.1: A sample message set, and examples of received messages with noise

message, distorted by noise in the channel. Assuming we were at the receiver and could observe these received points as they arrived, what would be good strategy for determining whether message A or B was transmitted? We might decide to bisect the plane along the $y$ axis and rule that received messages on the left side of the plane were transmitted as message A, and those on the right side of the plane were transmitted as message B. Assuming message $\mathrm{A}$ and $\mathrm{B}$ are transmitted with equal likelihood, this turns out to be the optimal strategy. However, when looking at the graph, we can see there are cyan circles that lie to the right of the $y$-axis as well as magenta dots that lie to the left. These are instances where our 
decoding scheme would fail and we would incorrectly assume message A was transmitted when message B actually was, or vice versa.

What Shannon proved for this channel was, even in the presences of random noise, one can achieve error-free communication up to a certain data rate. This maximum rate, known as the channel capacity, $C$, is the upper limit on the rate error-free communication can occur over the channel and depends only on the physical characteristics of the channel. Conversely, communication over the channel at a rate greater than $C$ cannot be error-free and the probability of communication errors will tend to $100 \%$ as the rate increases above $C$.

Unfortunately, Shannon's theory does not provide a scheme or technique for communication, but merely states strategies exist for error-free communication up to the channel capacity. It's obvious that the sample messages and decoding technique presented above are suboptimal, as transmission errors have already been encountered. Shannon's work provides a benchmark against which designers can compare communication systems for optimality.

\subsection{Signal to Noise Ratio and Channel Characteristics}

To further appeal to Shannon's channel capacity, we must analyze the parameters in 2.1). In a world without constraint we could transmit the source message $s$ with an infinite amount of power. Referring to the previous sample messages $(-1,0)$ and $(1,0)$, we could space them arbitrarily far apart on the coordinate plane, using $(-10,0)$ and $(10,0)$, or even $(-100,0)$ and $(100,0)$. Using this technique we could space the messages far enough apart that the random perturbation incurred during transmission has little impact.

As is often the case in engineering, this is not without cost. Energy is required to transmit messages, and in this case, a coordinate's distance form the origin is proportional to the energy necessary for transmission. For the purposes of this paper we won't be concerned with the energy of specific messages. We shall only be concerned with the average power of the set of messages.

For a given message set, we must quantify the average amount of power required to transmit messages. The average power for a given message set $X=\left\{x_{1}, x_{2}, \ldots, x_{n}\right\}$, as 
shown in [7], is

$$
P_{X}=\frac{1}{n} \sum_{i=1}^{n} x_{i}^{2} .
$$

In practice, systems are designed with a power-constraint. Even if a source is connected to an infinite energy source, an outlet for instance, transmission power is still finite. This is compounded for battery-powered devices, or devices designed for low power consumption. Given a power constraint $P$, a communication system must satisfy the inequality

$$
\sum_{i=1}^{n} x_{i}^{2}<P .
$$

As shown in figure 2.1, the power constraint and the power of the noise are at odds. In the AWGN channel model, the noise is considered "white." White noise has equal energy in all frequencies; it is not frequency selective. Examining a chunk of frequency, say $10 \mathrm{~Hz}$, anywhere along the noise spectrum, white noise will exhibit statistically equal power. Thus, when modeling white noise it is independent of the actual frequencies, and depends only the bandwidth. When modeling white noise, we assume a power spectral density $\frac{N_{0}}{2}$. The power spectral density, or PSD, is a measure of the energy density in terms of frequency. A more formal definition of $\frac{N_{0}}{2}$ is given in [8].

The channel capacity depends on the ratio of power available for transmission and the power of noise in the channel. This ratio is called the signal-to-noise ratio, or SNR. Shannon presented the channel capacity for the band-limited Gaussian channel with noise PSD $\frac{N_{0}}{2}$ watts/Hz, transmit power $\mathrm{P}$ watts and bandwidth $W \mathrm{~Hz}$, shown below,

$$
C=W \log \left(1+\frac{P}{N_{0} W}\right) \text { bits/sec. }
$$

It is common to normalize the capacity by the bandwidth, commonly called the spectral efficiency

$$
\frac{C}{W}=\log \left(1+\frac{P}{N_{0} W}\right) \text { bits } / \mathrm{sec} / \mathrm{Hz}
$$

\subsection{Summary}

In this section we've introduced an additive white Gaussian noise model for a communication system. Several graphical examples were given to help illustrate the process of 
transmitting messages across an AWGN channel, and the noise that occurs during transmission. We've presented power-constraints that are present in any practical system, and how the signal-to-noise-ratio, or SNR, is a metric used to represent the power constraint in relation to the noise present in the system.

In addition, we introduced Shannon's concept of channel capacity. Shannon showed that over an AWGN channel a transmission rate can be achieved with near-zero probability of error. Unfortunately, Shannon's work does not provide a strategy for achieving this rate, but only states that a strategy does exists. 


\section{Chapter 3}

\section{Wireless Fading Channels}

\subsection{Introduction to the Wireless Channel}

In the previous chapter we spoke only of the AWGN channel. AWGN is a good model for wired communication, and more general AWGN models work well for certain types of wireless communication such as infrared. However, the general wireless medium presents challenges that are not present in the AWGN model. Signal power degrades much faster in free-space transmission than it does through a copper wire. When communicating over a wire signal energy is guaranteed to arrive at the destination, although attenuated. We are afforded no such luxury in the general wireless medium.

Mobile phone users have some familiarity with the effects of the more hostile environment. Wireless communication systems must deal with underground tunnels that limit connectivity and mountains or other large geographic features that obstruct transmission. In addition, the wireless bandwidth is not a dedicated resource. In a point-to-point medium the transmitter need not worry about interference from other transmitters. However, wireless devices operate in a broadcast environment, and devices within range of each other must compete for use of the spectrum.

The wireless environment is hostile, and presents more challenges than the AWGN model, but the appeal of mobile units and freedom from wires is very alluring. The information presented here will help form a foundation for the work we present later in the thesis. The remainder of this chapter will present the challenges of the wireless channel in more detail, 
as well as quantitative ways of describing them.

\subsubsection{Fading Components}

\section{Path Loss and Shadowing}

When transmitting wireless messages over the air, there is no directed medium or waveguide as is the case with a wire. Transmission through free-space requires more power, and the case only worsens as distances increases. Transmission energy attenuates with the inverse square of the distance [9]. The attenuation of energy over distance is known as path loss. It should be clear that efficient use of a power budget is of paramount importance.

Shadowing is the random fluctuation in received signal strength. It is most often attributed to large-scale blockages in the wireless channel, such as mountains. While path loss may largely be deterministic, shadowing is dependent on the environment and must be modeled statistically. The combination of these effects lead us to a random, but statistically characterized model for the wireless channel.

\section{Multipath}

In addition to the previously mentioned effects, multipath provides another challenge to wireless communication. Typically, wireless transmitters use omnidirectional antennas. These antennas transmit signal power in all directions. As a result, transmissions travel in every direction and encounter any number of objects or reflectors. While the receiver may receive the line-of-sight transmission, it also likely receives reflected copies of the transmitted signal. In an urban environment a receiver will receive a line-of-sight transmission, but also many copies of the transmitted message reflected off the faces of buildings.

These reflections, or multipath components, are distorted in two ways. First, the messages all travel through separate pathways to the receiver and experience distinct attenuation. Also, the distance travelled will be longer than the line-of-sight link, and the messages will arrive at the receiver at later times. The multipath components will be out-of-phase with the line-of-sight transmission and with each other. They will interfere with themselves and the line-of-sight transmission constructively and destructively. As a result, the destination will 
receive the sum of all these message that may not resemble the transmitted message. Often a line-of-sight link is unavailable and the receiver may only be able to rely on the multipath components.

\subsubsection{Fading and the Gaussian Model}

Recall (2.1). Using this model, the transmitted message is corrupted only by additive Gaussian noise. An extension of this model would be to provide a coefficient of attenuation,

$$
y=a s+n .
$$

In the extension of the AWGN channel, this coefficient is a complex number with magnitude typically less than unity representing attenuation due to line loss, and a phase shift. The coefficient may even be a function of frequency, $a(f)$. However, these characteristics are typically deterministic and are known to the receiver.

Unfortunately, in the wireless channel both the magnitude and phase of this coefficient are random, and $a$ is commonly called the fading coefficient. For the moment, assume this coefficient has magnitude one. In this scenario, the strength of the transmission will be unaffected by the channel. We'll assume the phase of the coefficient varies randomly, uniformly distributed between 0 and $2 \pi$ radians. We'll also ignore effects of the additive noise.

As figure 3.1 shows, even without attenuation the task at the receiver is much more difficult than in the AWGN case. Figure 3.2 shows the effects of both fading and additive noise. Unfortunately, using the previously presented decoding scheme, both of these channel yield disappointing results.

\subsubsection{Channel Statistics}

Given the effects of fading during transmission, we desire strategies for combatting it. The strategy used depends on what the transmitter and receiver know about the wireless channel. When the transmitter can accurately measure the forward-channel to the receiver, it can minimize the effects of fading by adapting the transmitted message. When the receiver 


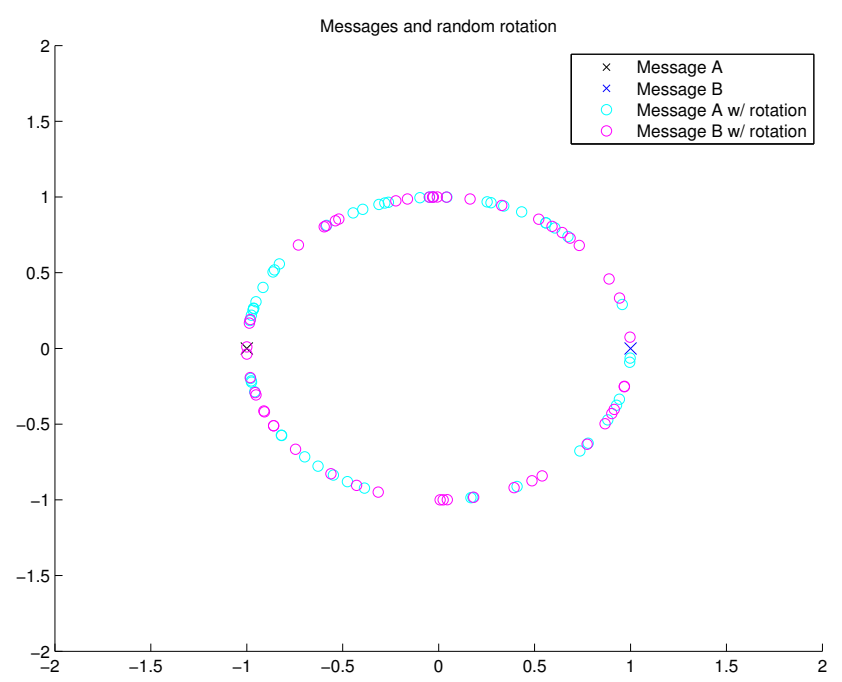

Figure 3.1: Messages distorted by random rotation

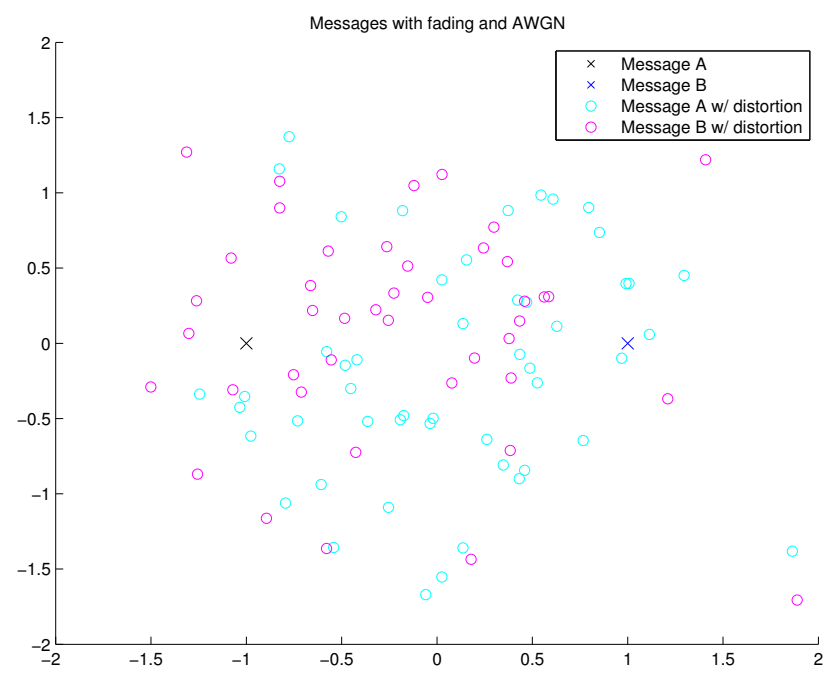

Figure 3.2: Messages distorted by fading and additive noise

can measure the channel but the transmitter cannot, it can also reduce the effects of fading. These factors influence the assumptions we make when modeling a system, and are presented below briefly.

\section{Channel Knowledge Available to Receiver}

When the receiver can accurately measure the channel state (fading coefficient), it can also take steps to improve decoding performance. For instance, if the receiver can accurately 
measure the fading coefficient it can remove the phase distortion incurred during transmission.

\section{Channel Knowledge Available to Transmitter and Receiver}

With channel knowledge available at the transmitter and receiver, the transmitter can adapt transmission strategies as the forward channel varies. For instance, the transmitter could increase it's transmission power when the channel conditions are poor and decrease power when the channel is more favorable. While intuitive, this is a suboptimal strategy, and it is better for the transmitter to remain silent when the channel state is below a certain threshold, and only transmit when channel conditions are favorable [9].

\subsection{The Rayleigh Fading Model}

To analyze the random effects of fading we must characterize it statistically, much like AWGN. When considering a fading model, we must determine whether the system is wideband or narrowband. If the inverse of the message bandwidth $B$ is significantly greater than the delay spread of the channel $T_{m}$ then we model the system as a narrowband fading channel. In other words, if $T_{m}<<B^{-1}$, then the channel is a narrowband fading channel. If $T_{m}>>B^{-1}$ then the channel is a wideband fading channel.

\section{Wideband Fading}

In a wideband model, messages will experience frequency selective fading. Under wideband fading, the message bandwidth $B$ is much larger than the coherence bandwidth $B_{c}$ of the channel. The coherence bandwidth $B_{c}$ is the bandwidth over which the channel response is correlated, or flat. In other words, if $B>B_{c}$ then the transmission will experience a channel response that varies across the transmission bandwidth, $B$.

\section{Narrowband Fading}

In a narrowband model, $B<<B_{c}$. This scenario is the opposite of the wideband model. When the transmission bandwidth is much less than the coherence bandwidth of the channel, 
the transmissions will experience a flat frequency response through the channel. Narrowband fading is sometimes called flat fading.

\section{Rayleigh Fading}

In a wireless environment with many reflectors and no strong line-of-sight component the fading coefficient may be modeled as the sum of two uncorrelated Gaussian processes. The assumption of Gaussian distribution is justified by appealing to the central limit theorem. If there are a large number of multipath components arriving at the receiver with random amplitude and phase, the distribution resulting from the sum of these components is approximately Gaussian [9].

Given two Gaussian random variables $X$ and $Y$ with zero mean and equal variance $\sigma^{2}$, the magnitude of the sum is $z=|a|=\sqrt{X^{2}+Y^{2}}$. This random variable, the envelope of the received signal, $z$, will have a Rayleigh distribution. The Rayleigh distribution is characterized by the probability density function (PDF)

$$
p_{z}(z)=2 \lambda z \exp \left(-\lambda z^{2}\right), \quad z \geq 0,
$$

or cumulative distribution function (CDF)

$$
P_{z}(z)=1-\exp \left(-\lambda z^{2}\right), \quad z \geq 0
$$

where $\lambda=\frac{1}{2 \sigma^{2}}$.

Figure 3.3 shows a Rayleigh PDF with parameter $\lambda=5$. Looking at the PDF, we see with high likelihood the fading coefficient magnitude will be much less than unity. In this figure, using the CDF in (3.3), the probability the fading amplitude is less than half is $P_{z}(0.5)=1-\exp (-1.25)=71.4 \%$.

We also consider the distribution of $|a|^{2}$, as it affects the SNR at the receiver. If $a$ is a Rayleigh random variable with parameter $\lambda=\frac{1}{2 \sigma^{2}}$ then $|a|^{2}$ is an exponential random variable. The PDF of an exponential random variable with parameter $\lambda$ is

$$
p_{a^{2}}(x)=\lambda x e^{-\lambda x}, \quad x \geq 0,
$$

and the $\mathrm{CDF}$ is

$$
P_{a^{2}}(x)=1-e^{-\lambda x}, \quad x \geq 0 .
$$




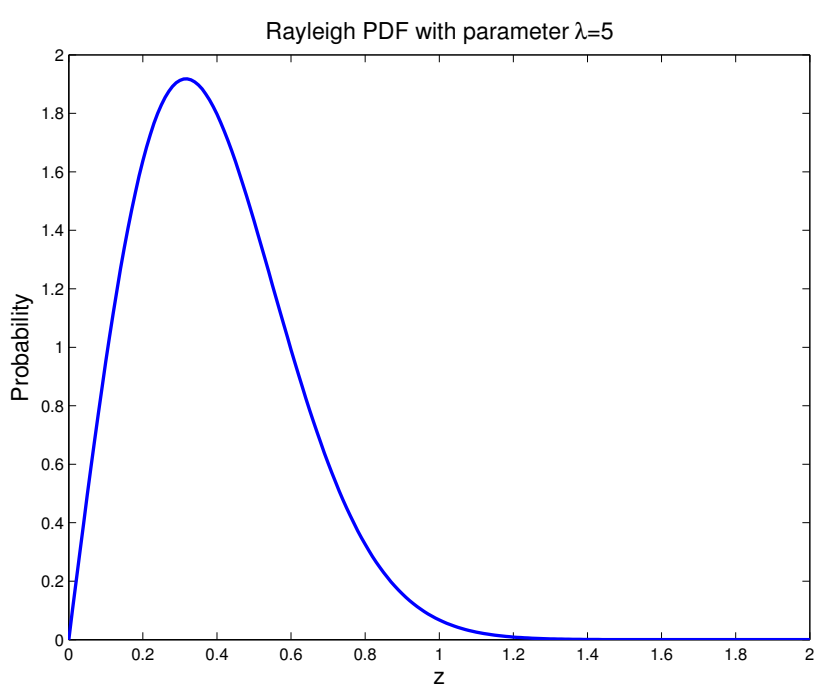

Figure 3.3: Rayleigh PDF with parameter $\lambda=5$

\subsection{Diversity}

With a hostile wireless channel, communication system designers must use techniques that aren't necessary in non-fading channels. A technique that is useful is diversity. Diversity employs multiple paths through the wireless channel. While it may sound abstract, these paths may be through time, space or frequency. For instance, in a wideband fading scenario, while the channel response for one frequency range may be unfavorable, another chunk of frequency may exhibit more promising quality. In fact, both OFDM and CDMA technologies exploit this quality. In the following paragraphs we'll introduce three types of diversity; time diversity, space diversity and cooperative diversity.

\section{Time Diversity}

Time diversity exploits the varying nature of the wireless channel. There are several ways to think about this. First, consider a scheme where the source wishes to transmit a message to the destination. One approach is to repeatedly transmit the message a large number of times. Depending on the variation of the channel, by repeatedly transmitting the same message it's much more likely that one of the transmissions may experience a favorable channel and be decoded at the receiver. 
Repetition, while plausible, is not very efficient as the same message must be transmitted repeatedly, keeping the system from transmitting any new messages. In practice, a technique called interleaving is often used. When interleaving, a transmitter typically has multiple messages to send that have been encoded using forward error correction, FEC. These messages are immune to small perturbations in their actual contents, and can withstand some amount of distortion. The transmitter slices each message up, intermixing the portions of each message. The order that the messages are intermingled is known in advance at the receiver, such that it can reconstruct each message from the interleaved incoming message.

If the channel experiences a poor condition for a short amount of time then a small portion of all messages will be corrupted. However, if the poor channel condition lasts for a sufficiently small amount of time the amount of corruption to each message will be minimal, and hopefully all of the original messages have received a small enough distortion to be recovered at the receiver.

For a system to take advantage of time diversity, the receiver must experience many channel realizations during a transmission. In other words, the channel quality must vary while a message, or messages, is being sent. This is called fast fading. In a slow fading environment, messages are transmitted in a short enough time period that the receiver experiences essentially a constant channel quality. Under slow fading a receiver can not employ time diversity techniques.

\section{Space Diversity}

For a system to utilize space diversity, it must employ multiple transmit or receive elements. Typically, this is done using multiple transmit or receive antennas at the source or destination respectively. Under specific space constraints, namely that each antenna be spaced at least one-half wavelength apart [9], the messages received at the destination will experience distinct channel realizations. When using multiple antennas, the messages transmitted or received from different antennas will travel different physical paths to the destination. The receiver can then use several techniques for combining the incoming messages to maximize the utility of "good" channels. 


\section{Cooperative Diversity}

Cooperative diversity is a special type of space diversity. Cooperative diversity considers more than a source and a destination. In a cooperative scenario we assume there are other wireless nodes in the network. When the source transmits a message intended for the destination, other nodes in the network will overhear the transmission. These nodes cooperate to assist in forwarding the message on to the destination. In the following chapter cooperative diversity will be visited in more detail.

\subsection{Summary}

In this chapter we presented the Rayleigh fading model. Several examples were provided that highlight the difficulties in designing a communication system for this environment. Channel state knowledge assumptions at the transmitter and receive were presented as they impact the development of the system model. The concept of diversity was introduced and types of diversity were discussed. 


\section{Chapter 4}

\section{Spatial Diversity and Cooperation}

\subsection{Overview}

As we've seen, the wireless fading channel is much more hostile than the AWGN channel. The nature of the wireless medium is inherently different than that of a non-fading medium, and novel techniques must be developed to improve performance. Past research has leveraged the broadcast nature of the wireless channel. By employing multiple antennas at the transmitter or destination, with the antennas spaced sufficiently far apart, transmissions will pass through several independent spatial paths. While one of these paths may experience poor channel conditions, it is less likely that all paths are exhibiting the same poor channel conditions simultaneously. Exploiting these multiple copies of messages is known as spatial diversity.

\subsubsection{Multiplexing}

Before proceeding, we should note that independent channels between the source and destination can be used for diversity, but may also be used to achieve multiplexing. Since the pathways between source and destination are independent, the messages sent along these distinct pathways will not influence each other. Therefore, it is possible for the source to transmit independent messages along these pathways, increasing the amount of data being transmitted simultaneously. This was first shown in [10].

However, as was described in chapter 3 , the independent transmission pathways achieved 
via space diversity can also be used to transmit multiple copies of the same message. Intuitively, we can think of diversity and multiplexing as using the same resource. In [11] it was shown that diversity and multiplexing are inherently linked. If a designer is using the resources of the channel optimally, diversity can only be improved at the cost of multiplexing, and vice-versa.

\subsection{Spatial Diversity}

\subsubsection{Relaying}

The relay channel consists of a source $s$, a relay $r$ and a destination $d(s)$. In the relay channel, $s$ transmits messages intended for $d(s)$. During this process, $r$ will overhear transmissions from $s . r$ will then attempt to assist $s$ in transmitting the message to $d(s)$. Capacities for the relay channel were first explored by Cover and Gamal in 1979 [12].

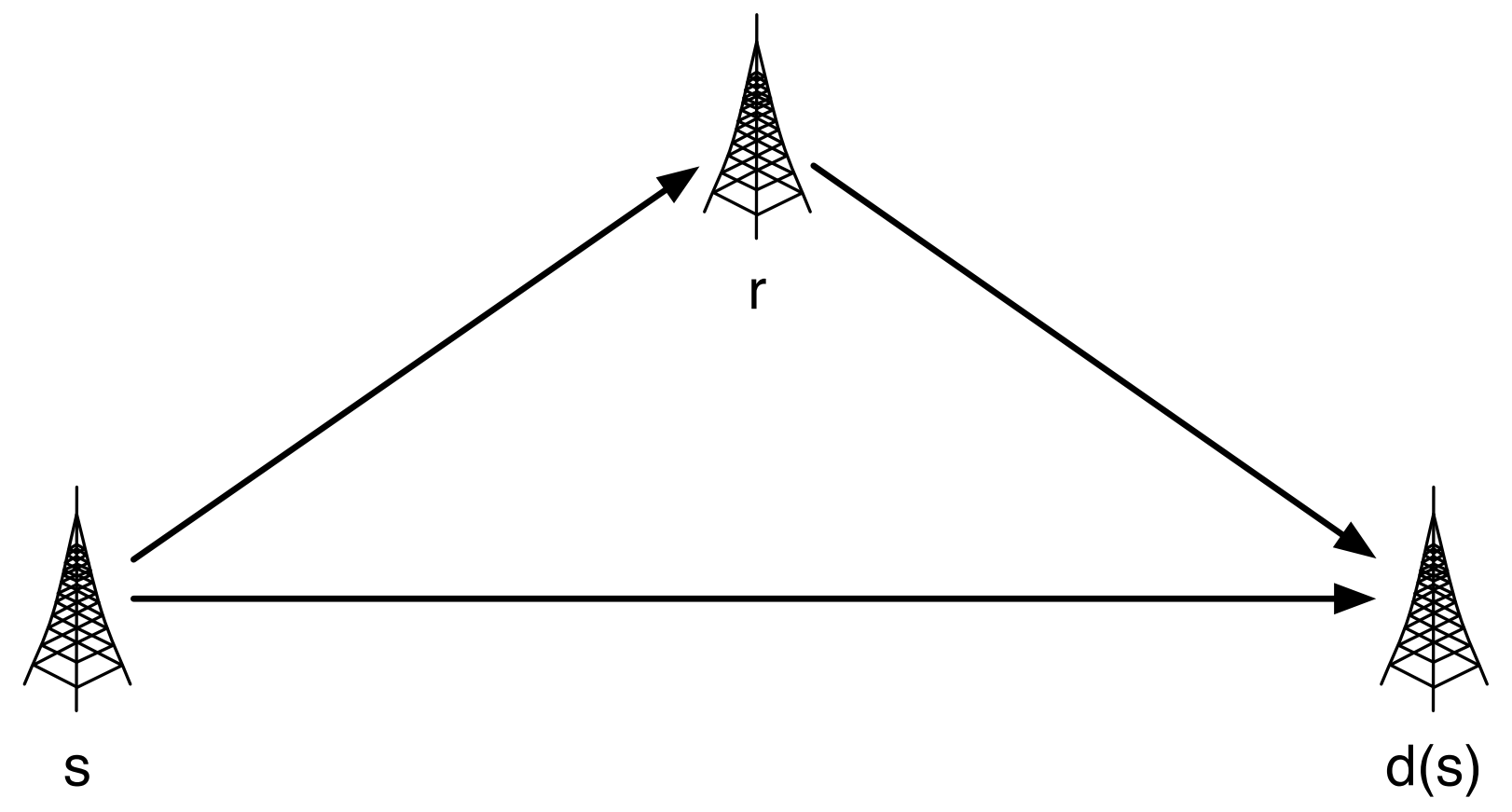

Figure 4.1: A relay network consisting of a source, s, a relay, r, and a destination $\mathrm{d}(\mathrm{s})$. 


\subsubsection{MIMO}

The benefits of multiple antennas at the receiver and destination were first highlighted by Winters [13], and later more thoroughly explored by Foschini and Foschini and Gans, respectively [10], [1]. For a communication system with $M_{t}$ transmit antennas and $M_{r}$ receive antennas, it has been shown that the maximum achievable diversity is $M_{t} M_{r}$. The maximum achievable multiplexing gain is $\min \left(M_{t}, M_{r}\right)$. Zheng and Tse showed additionally that a MIMO system can achieve combinations of diversity and multiplexing between these points dictated by a curve known as the diversity-multiplexing tradeoff (DMT) curve [11]. However, the aforementioned maximum diversity and multiplexing values are the extremes, and one can only be improved by sacrificing performance from the other.

The above research proved multiple antennas at the transmitter or receiver, or both, could substantially improve the performance of a wireless network. In addition, the work showed that system designers can trade-off diversity benefits for multiplexing benefits. However, for multiple antennas systems to provide transmit pathways that experience independent fading, they must be separated by at least one-half wavelength of the carrier frequency [9].

\subsubsection{Cooperative Diversity}

For small wireless devices that operate at high-frequency, antenna arrays on the device are impractical. Consider a mobile phone that operates in the $900 \mathrm{MHz}$ spectrum. Recalling the speed of light is $c=3 \times 10^{8}$ meters/sec, the wavelength of a $900 \mathrm{MHz}$ electromagnetic wave traveling through the atmosphere is $\lambda=\left(3 \times 10^{8}\right) /\left(900 \times 10^{6}\right)=0.33$ meters, or approximately one foot. For mobile phones operating at this frequency, multiple antennas would need spaced 0.15 meters, about 6 inches apart. These spacings are impossible to achieve on devices that are themselves often smaller than the required antenna spacing.

While MIMO is attractive, there are classes of wireless networks that simply cannot take advantage of the benefits. While multiple antennas provide a performance increase for a given power budget, additional cost is incurred to manufacture units with multiple antennas. When implementing MIMO, additional receiver or transmitter processing and hardware may be required in addition to the antennas themselves. For applications requiring 
low-cost wireless nodes, for instance sensor networks, this additional cost is prohibitive.

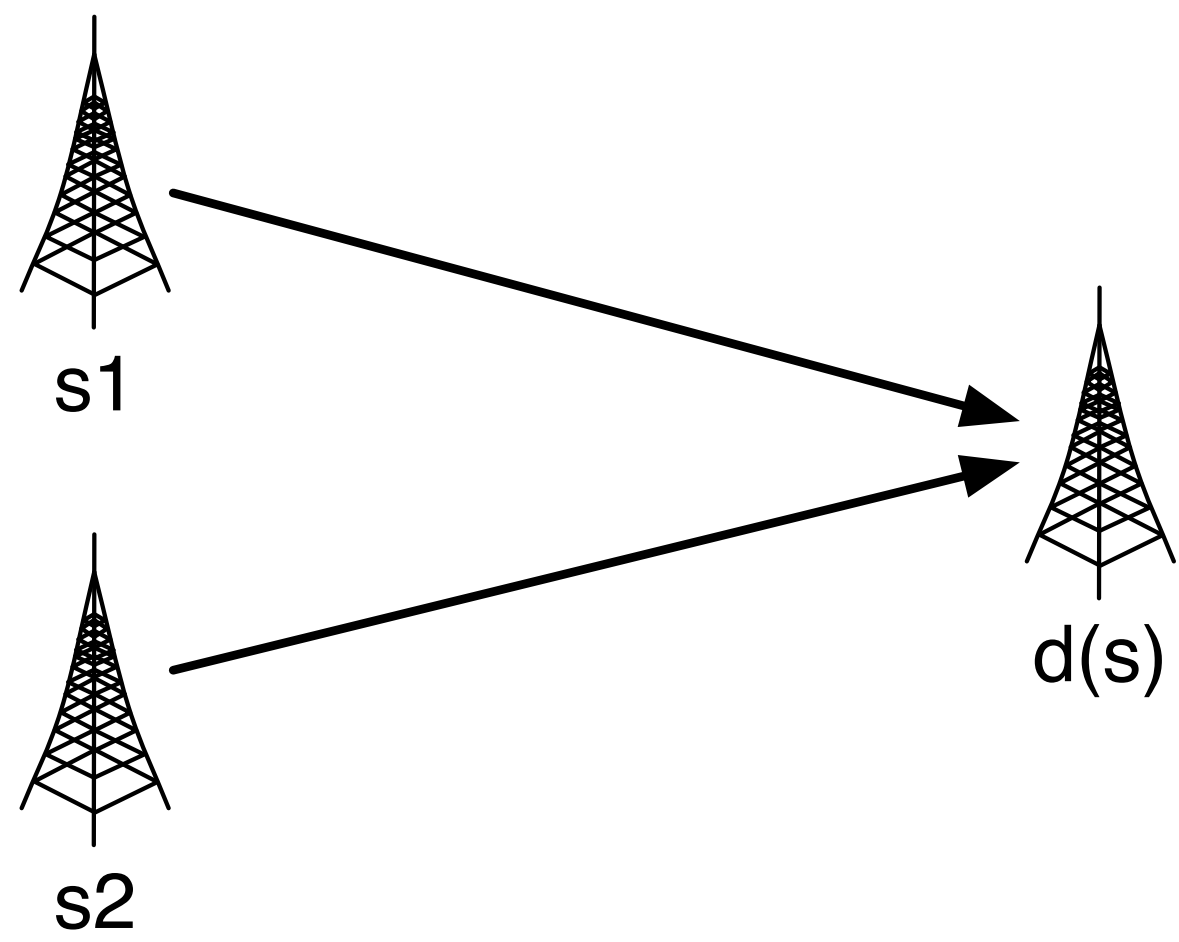

Figure 4.2: A two-transmitter network. $s_{1}$ and $s_{2}$ are two transmitters attempting communication with $d(s)$.

Wireless transmissions are broadcast in nature. As we've seen, since the transmit energy is not directed, the signal deteriorates much quicker than a point-to-point link. However, this means that other wireless receivers may be able to overhear a transmission. These other receivers can then cooperate to forward messages on to the destination. Using this technique multiplexing gain cannot be achieved, but gain in diversity can.

Consider a wireless network with two transmitters, $s_{1}$ and $s_{2}$, both transmitting messages in separate time-slots intended for the same destination, $d(s)$. In the first time-slot $s_{1}$ transmits while $s_{2}$ remains silent, and in the second time-slot $s_{2}$ transmits while $s_{1}$ remains silent. During $s_{1}$ 's transmission, $s_{2}$ will receive a noisy version of $s_{1}$ 's transmission. Also, in the second time-slot $s_{1}$ will receive a noisy version of $s_{2}$ 's transmission. In a non-cooperative system this information is simply ignored or discarded. However, it is possible for $s_{1}$ and $s_{2}$ to forward the information overheard to the destination. 
User cooperation, or cooperative diversity, was first presented in [14] and [15. Sendonaris et. al. showed that as a result of cooperation users experienced better rates and more reliable communication. When designing a cooperative network, nodes typically employ one of two forwarding strategies: amplify-and-forward (AF) or decode-and-forward (DF).

\section{Amplify and Forward}

Using amplify-and-forward, relays overhear a noisy message from the transmitter. The relay does not attempt to decode the message, but rather simply amplifies the received message up to it's power constraint and retransmits the amplified messages. AF lends itself to a straightforward analysis. However, by nature of the amplification, noise in the original transmission is propagated and the destination must have knowledge of the inter-user channel for optimal decoding.

\section{Decode and Forward}

Under decode-and-forward relays attempt to decode the information received from another node. Then, the relaying node will re-transmit the message. Assuming the relay correctly decodes the message, the noise of the original transmission will be removed and will not be propagated. Unfortunately, if the message is decoded incorrectly the relay will transmit a message that is not identical to the source message which will be detrimental to decoding at the destination. Laneman and Wornell proposed a protocol where relays choose to aid in cooperation dependent on whether the source message was correctly decoded by monitoring the channel state [16]. 


\section{Chapter 5}

\section{Multimodal Cooperation Using Repetition}

Considering the difficulties when communicating in a wireless environment, and the inability of a purely wireless network to scale, we present a cooperative protocol for a multimodal wireless network. While hybrid networks currently exist, for example cell networks have wireless mobiles as well as base stations with both wired and wireless capabilities, these modes are optimized separately and then joined. We propose optimizing power allocation across both modes simultaneously, a multimodal network. Hybrid networks often require little additional hardware to function as a multimodal network.

In this section, we describe the addition of a non-fading communication mode, e.g. a wire, to a cooperative wireless network. We base our protocol on that originally presented in [16]. We rely on information outage probability to analyze the performance of an additional non-fading mode placed between a.) a transmission source and a relay, and b.) a relay and the destination.

\subsection{Network Structure}

We assume a network of $\mathcal{M}=\{1,2, \ldots, m\}$ transmitters and a destination, $d(s)$. Each of $m$ the transmitters has its own information to transmit to $d(s)$. The transmitters all operate within the same wireless bandwidth, $B_{w l}$. We assume each node has continuous-time power 
constraint of $P_{c}$ joules per second $(\mathrm{J} / \mathrm{s})$.

\subsection{Medium Access Control}

\subsubsection{Degrees of Freedom}

Before describing the medium access schemes used, we must first introduce the term degrees-of-freedom (DOF). The degrees-of-freedom for a communication channel are essentially the resources available for transmission. In this work we consider the wireless bandwidth and the time available for transmission as the resources available, or the timebandwidth product. A visualization of the degrees of freedom is shown in figure 5.1. The definition of DOF for a wireless channel was described in-depth in [11.

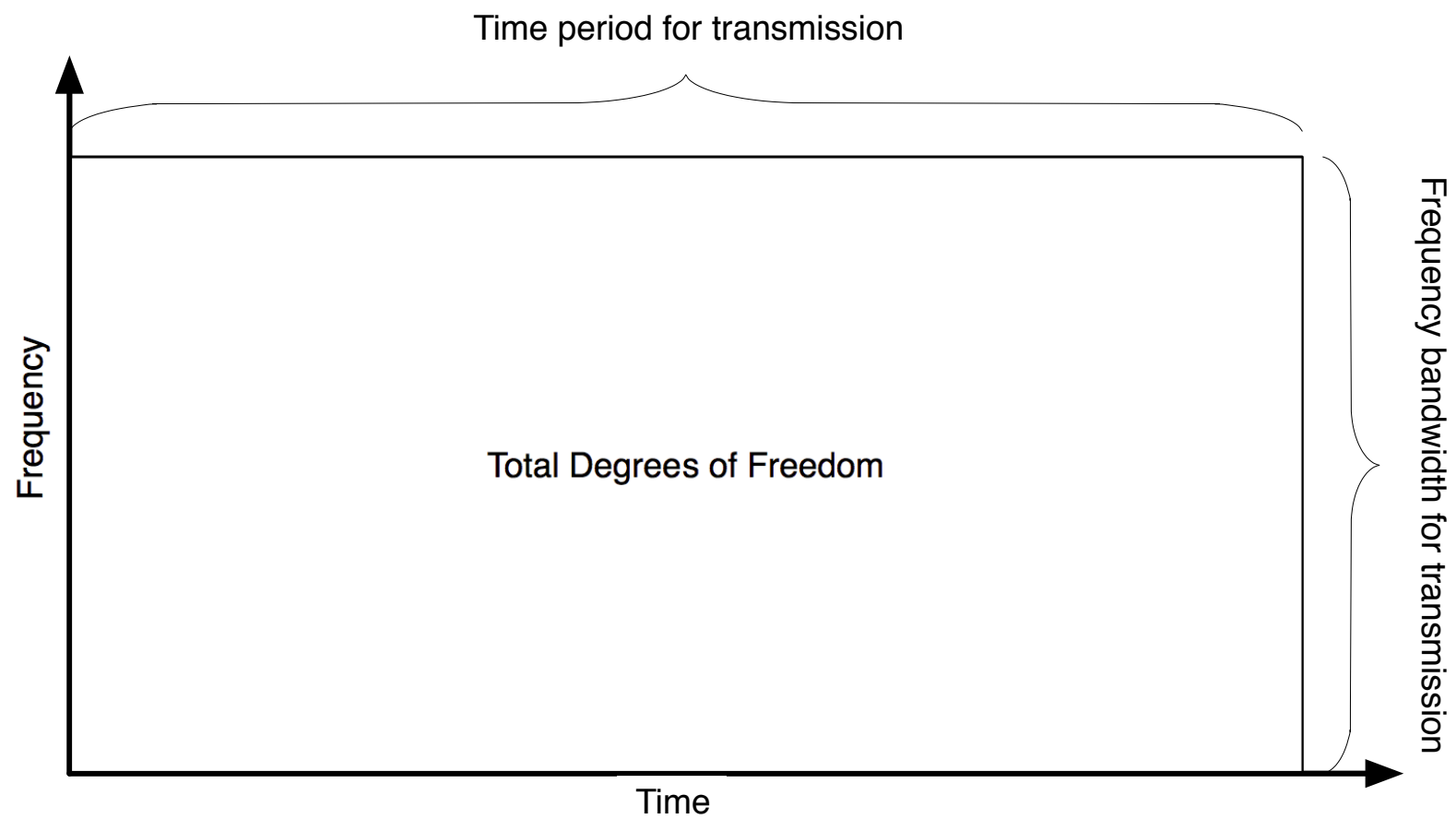

Figure 5.1: The time-bandwidth product degrees-of-freedom 


\subsubsection{Noncooperative Transmission}

To highlight the transmission schedule for each device, we contrast with a traditional non-cooperative transmission strategy. Under this strategy, each transmitter is assigned non-interfering, orthogonal channels. Each transmitter can transmit the entire time period. This strategy is illustrated below in figure 5.2. Nnote that this is a half-duplex system. No node is simultaneously transmitting and receiving in the same channel. This will be true for all the medium access control schemes discussed in this thesis.

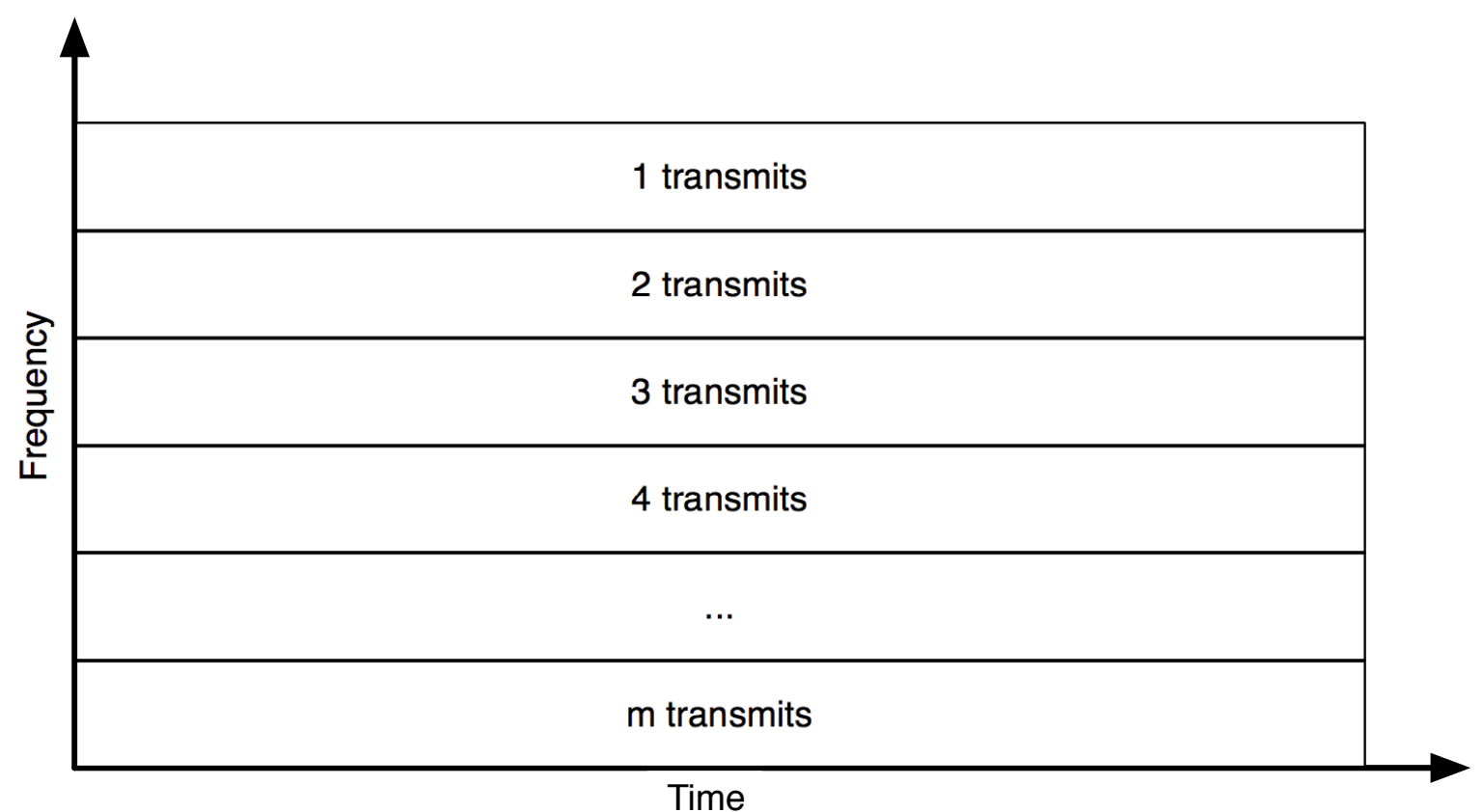

Figure 5.2: Medium access control for noncooperative communication

\subsubsection{Repetition Cooperation}

Using a repetition-based cooperative strategy, the wireless bandwidth is again separated into non-overlapping channels. However, each transmitter does not transmit for the entire time period. Instead, each channel is also divided into $m$ equivalent time periods. In the first time-slot, each of the $m$ nodes transmit in their own channel. For each of the subsequent time periods, assuming received messages are decoded, each of the $m$ transmitters relays 
overheard transmissions as shown in Figure 5.3. Each transmission occurs in $1 / \mathrm{m}^{2}$ of the DOF

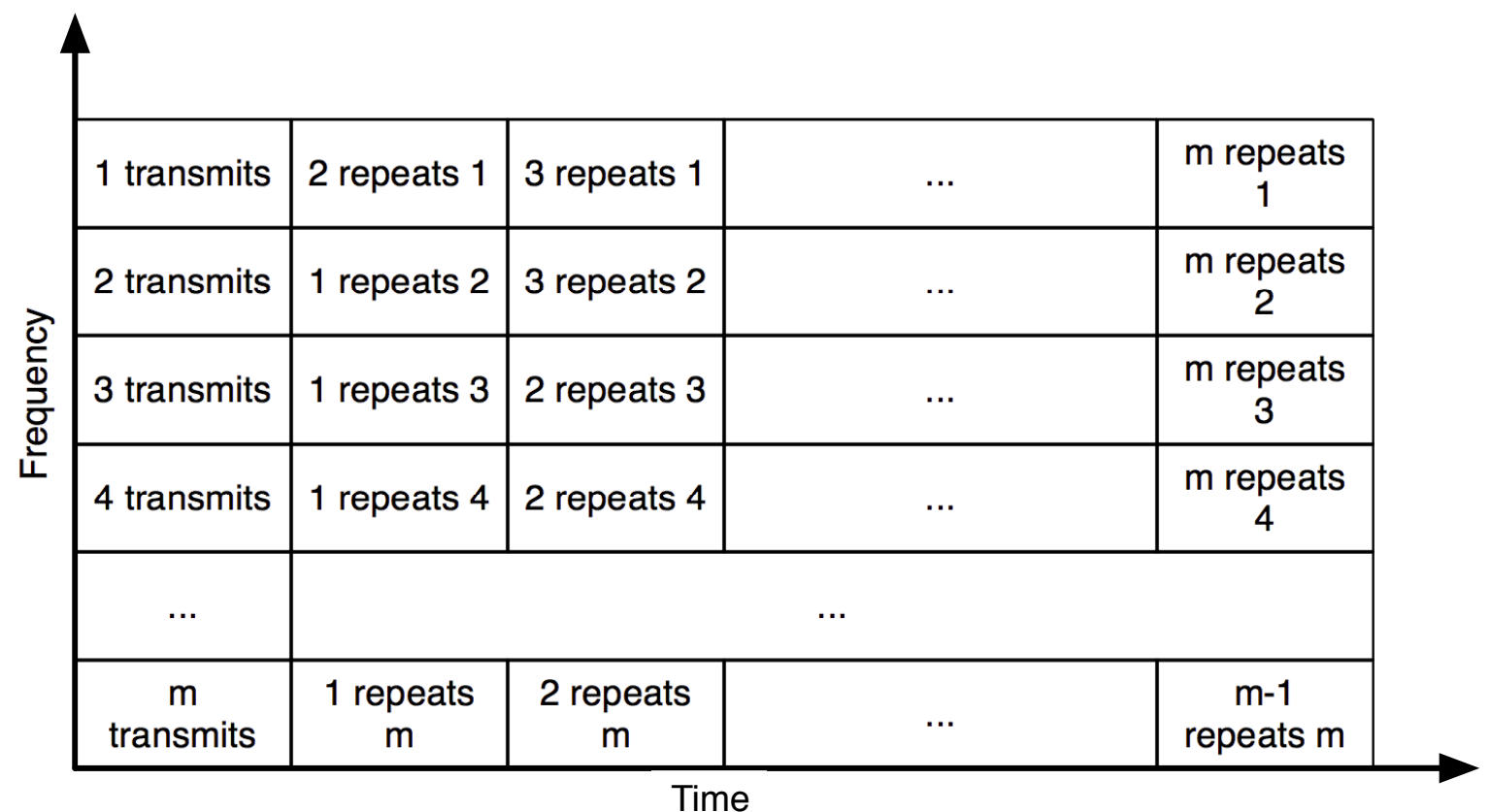

Figure 5.3: Medium access control for cooperative repetition communication

\subsection{System Model}

We focus on the scenario where a single node, $s$, is transmitting to a destination $d(s)$. Since our network consists of $m$ transmitters, every transmitter except the source is available to relay, hence there are $m-1$ relays, as shown in figure 5.4. The source-relay links and relay-destination links are considered, as well as the source-destination direct link. Inter-relay wireless channels are not used.

During the first stage of transmission, the source $s$ transmits. The relays receive a message of the form

$$
y_{r_{i}}[n]=a_{s, r_{i}} x_{s}[n]+z_{r_{i}}[n], \quad 1 \leq i \leq m-1,
$$

where $y_{r_{i}}[n]$ is the received message at relay $r_{i}, a_{s, r_{i}}$ is the fading coefficient between $s$ and 


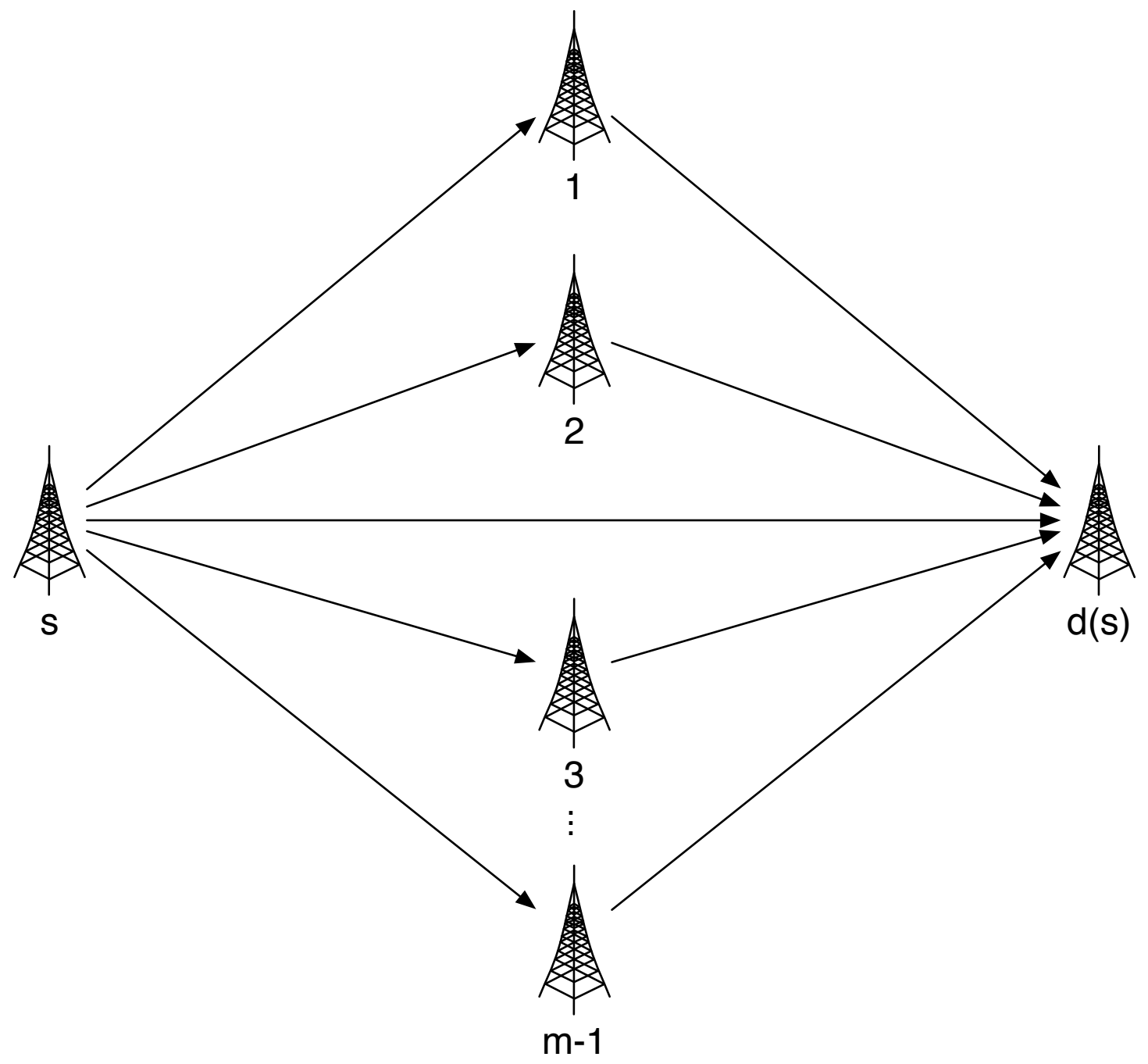

Figure 5.4: A cooperative network with source $s$ transmitting messages to destination $d(s)$ with $m-1$ relays available for cooperation.

relay $r_{i}, x_{s}[n]$ is the message transmitted by $s$ at time $n$, and $z_{r_{i}}[n]$ is the noise present at relay $r_{i}$ at time $n$.

We assume that each channel experiences independent, slow, flat Rayleigh fading. In other words, we assume the fading between pairs of nodes occurs independently and is constant across both the bandwidths of interest and the transmission time period. Thus, during a transmission the fading is time and frequency invariant.

The fading coefficients $a_{s, r_{i}}$, are modeled as Rayleigh random variables with parameter $\lambda_{s, r_{i}}$. Each wireless fading channel is characterized by a unique parameter, thus $\lambda_{s, r_{1}}$ param- 
eterizes the channel between $s$ and relay $r_{1}$ and corresponds to $a_{s, r_{1}}, \lambda_{m-1, d(s)}$ parameterizes the channel between relay $m-1$ and the destination $d(s)$ and corresponds to $a_{m-1, d(s)}$, and so on. Note that increasing $\lambda$ indicates increasing probability of coefficients of small magnitude. We assume $a$ includes the effects of path-loss and shadowing.

$z[n]$ is the thermal noise sample present at the receiver, which we model as zero-mean mutually independent, circularly symmetric complex Gaussian noise with variance $N_{0}$. In other words, we model the real and imaginary components (or in-phase and quadrature components) of the additive noise as independent Gaussian random variables of equal variance.

As shown in the previous chapters, for a fixed rate $R$, a receiver must experience a certain SNR to successfully decode messages transmitted at that rate. We now define the transmit $S N R$, or the SNR as defined without including the effects of fading. For a continuous-time channel with bandwidth $B_{w l}$ hertz used for transmission, this is equivalent to transmitting $B_{w l}$ two-dimensional symbols per second $(2-\mathrm{D} / \mathrm{s})$ in the discrete-time representation. If each node transmits under a continuous-time power constraint of $P_{c}$ Joules per second $(\mathrm{J} / \mathrm{s})$, this equates to $P_{c} / B_{w l}$ Joules per $2 \mathrm{D}$ symbol $(\mathrm{J} / 2-\mathrm{D})$. However, since each terminal is transmitting in only a fraction $1 / m$ of the degrees-of-freedom of the channel, then the power constraint becomes $P=m P_{c} / B_{w l} \mathrm{~J} / 2-\mathrm{D}$. Thus, the transmit signal-to-noise ratio, SNR, is defined

$$
\mathrm{SNR}=\frac{m P_{c}}{N_{0} B_{w l}}=\frac{P}{N_{0}}
$$

Again, (5.2) is the transmit SNR, SNR, and is based solely on the transmit power constraint and the noise. The actual SNR at a receiver will depend on the power of the fading coefficient, $|a|^{2}$. Thus, the SNR a receiver will experience will be SNR $|a|^{2}$, most likely a fraction of the actual transmit SNR. The received SNR will be a function of the quality of the channel, $\lambda$.

For a relay to decode at rate $R$ bits/sec, relay $r_{i}$ must experience an SNR such that $R<\frac{1}{m} \log \left(1+\operatorname{SNR}\left|a_{s, r_{i}}\right|^{2}\right)$, or $\left|a_{s, r_{i}}\right|^{2}>2^{m R}-1 /$ SNR. We assume that $R$ is set in advance, and that $r_{i}$ can accurately measure the received SNR, SNR $\left|a_{s, r_{i}}\right|^{2}$, to decide if it can decode. If the relay can decode it will relay the message $x_{s}[n]$ in the appropriate timeslot. If it cannot decode it will remain silent during the timeslot. 
Depending on the instantaneous channel conditions, we now see the set of relays that will successfully decode and assist in transmission will be a random set. Specifically, this set will be a subset of all available relays, $\mathcal{M}=\{1,2, \ldots, m-1\}$. We denote the random subset the decoding set, $\mathcal{D}(s)$. The ability for the desintation $d(s)$ to decode will depend on this random set.

During the initial transmission, the destination receives the source transmission via the direct-link, $y_{d(s)}[n]=a_{s, d(s)} x_{s}[n]+z_{d}(s)[n]$. For every relay that is able to decode the destination also receives the relayed transmission $y_{d(s)}[n]=a_{r_{i}, d(s)} x_{r}[n]+z_{d(s)}[n], \quad r_{i} \in \mathcal{D}(s)$. For purposes of simplicity, we assume $d(s)$ knows which relays successfully decoded and ignores any timeslots for relays that cannot decode. It is also important to note that the $z_{d(s)}[n]$ are all unique, independent realizations of the noise present at $d(s)$.

\subsection{Outage Probability Without Additional Modes}

In the equations below, we'll be referring to the probability that a random variable falls below a certain threshold, $\operatorname{Pr}[X<x]$. This is equivalent notation for the previously introduced $\mathrm{CDF}$ of $X$. So, $\operatorname{Pr}[X<x]$ is equivalent to $P_{X}(x)$.

The destination, $d(s)$, will receive the direct-link transmission, as well as relayed transmissions for $r_{i} \in \mathcal{D}(s)$, or for all relays that were able to decode the source transmission. In our system, the destination will add the signals together from all these transmissions. Assuming a decoding set $\mathcal{D}(s)$, the realized mutual information at the destination, I, will be

$$
\mathbf{I}=\frac{1}{m} \log \left(1+\operatorname{SNR}\left|a_{s, d(s)}\right|^{2}+\operatorname{SNR} \sum_{r \in \mathcal{D}(s)}\left|a_{r, d(s)}\right|^{2}\right) .
$$

The term $1 / m$ is a normalization of mutual information by the degrees-of-freedom in the channel. Each relay transmits for a fraction $1 / m$ of the degrees-of-freedom, so the mutual information I must be normalized as such.

In [16], it was shown that the system model presented above achieves the outage probability

$$
\operatorname{Pr}[\mathrm{I}<R] \sim\left[\frac{2^{m R}-1}{\mathrm{SNR}}\right]^{m} \times \sum_{\mathcal{D}(s)} \lambda_{s, d(s)} \times \prod_{r \in \mathcal{D}(s)} \lambda_{r, d(s)} \times \prod_{r \notin \mathcal{D}(s)} \lambda_{s, r} \times \frac{1}{(|\mathcal{D}(s)|+1) !} .
$$


Several interesting qualities are worth pointing out. From $(5.4)$, we can see the outage probability does not depend on instantaneous channel gains, simply the parameters describing the channel, $\lambda$. Second, the system achieves full diversity of order $m$, noted by the exponent of the scalar factor $\left(2^{m R}-1 / \mathrm{SNR}\right)^{m}$. This means the system takes advantage of all $m$ transmission pathways (the source $s$ and the $m-1$ possible relays). (5.4) depends only on on statistical parameters of the channel quality between nodes, the target rate, the number of transmitters $m$ and SNR. This will be important, as we can later interpret the addition of a non-fading mode as modifications to these parameters. Figure 5.5 plots (5.4) for several values of $m, R=1$ and $\lambda=1$ for all channels.

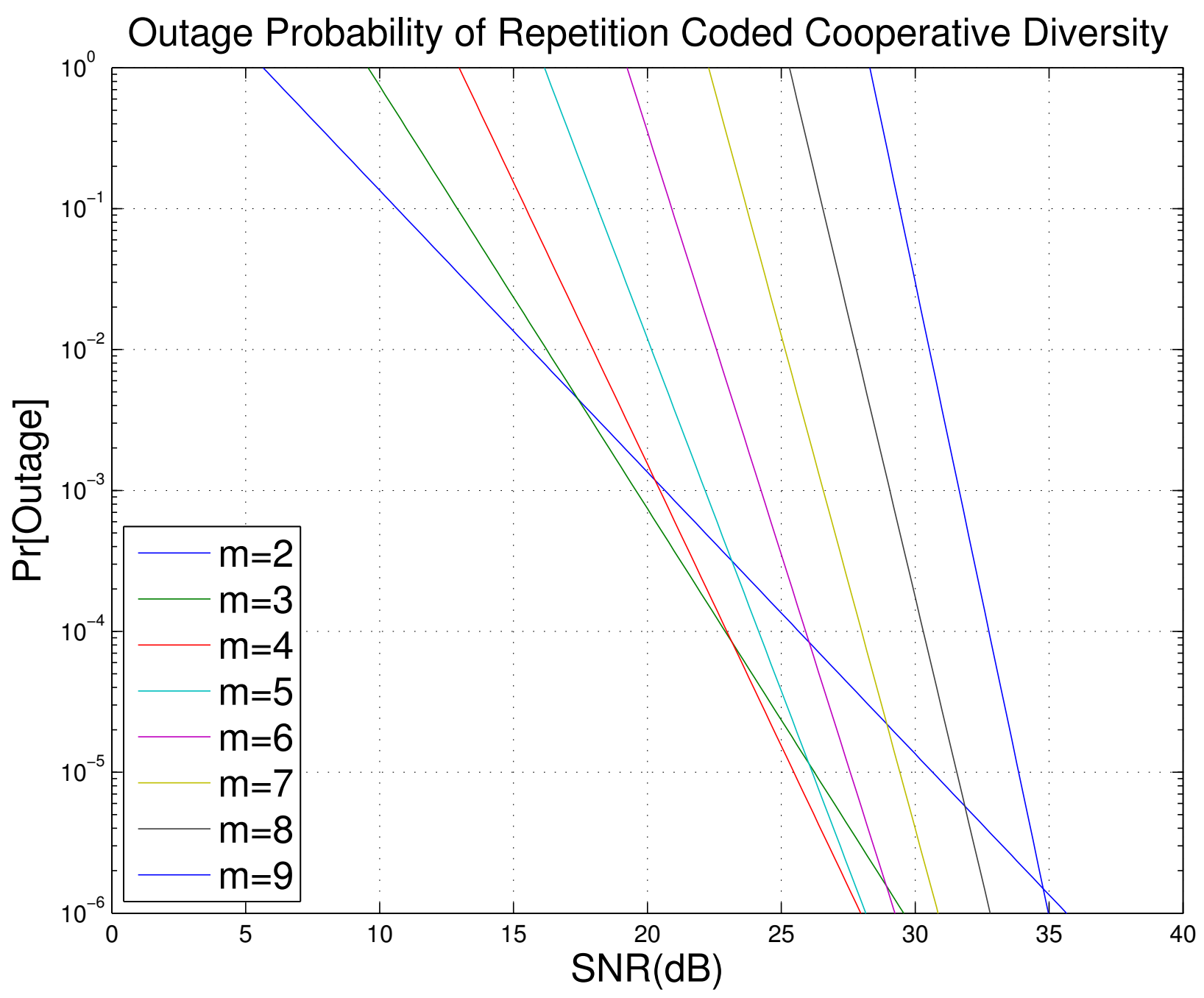

Figure 5.5: Repetition outage probability for a wireless network for several values of $m, R=1$, and $\lambda=1$ for all channels. 


\subsection{Adding a Non-Fading Mode}

We now consider the introduction of a non-fading mode of communication into the network. The additional mode will have a complex path gain $h$ and bandwidth $B_{w}$. We further assume $h$ is time-invariant and deterministic across the bandwidth $B_{w}$. The relay connected to this non-fading mode will be denoted $r_{w}$.

While another mode of communication is available, each node still has a finite power constraint. For any node transmitting through the additional non-fading mode, power must be divided between wireless transmission and transmission across the non-fading mode. We'll denote the fraction of power dedicated to non-fading transmission as $P_{w}$, where $P_{w} \in[0,1]$. Conversely, the fraction of power used for wireless transmission will be $1-P_{w}$.

The receiver of transmissions across the non-fading mode will receive messages of the form

$$
y_{r_{w}}[n]=h x[n]+z_{r_{w}}[n] .
$$

Just as with the wireless link, we assume some noise at the receiver for the non-fading link. Assuming an equal noise variance of $N_{0}$, the transmit SNR for the non-fading link is

$$
\frac{P_{w} B_{w l}}{B_{w}} \frac{m P_{c}}{N_{0} B_{w l}}=\frac{P_{w}}{B_{w} / B_{w l}} \mathrm{SNR}
$$

We assume that any transmitter connected to the non-fading mode transmits on both modes simultaneously, thus the multiplicative factor $m$ is retained. In general, the bandwidths available to the fading and non-fading communication modes will be different, so SNR is bandwidth-normalized by the term $B_{w l} / B_{w}$. The last equality is simply a bandwidth normalized fraction of SNR, as one might intuitively expect. Again, this SNR does not include the effects of the channel. The actual received SNR for the receiver connected to the non-fading mode will be $\frac{P_{w}}{B_{w} / B_{w l}} \operatorname{SNR}|h|^{2}$.

The additional mode provides another channel to achieve an additional rate. Specifically, as shown in Chapter 2, the capacity of this additional mode is

$$
C_{w}=B_{w} \log \left(1+\frac{P_{w}}{B_{w} / B_{w l}} \operatorname{SNR}|h|^{2}\right) .
$$

Thus, any rate less than $C_{w}$ is achievable over this mode. 


\subsection{Outage Approximation for an Additional Source- Relay Non-Fading Mode}

Proposition 1: Let $\bar{\lambda}_{s, r}=\lambda_{s, r}$ for all $r \neq r_{w}$. Let $\bar{\lambda}_{s, r_{w}}=\left(2^{m \hat{R}}-1\right) /\left(2^{m R}-1\right) \lambda_{s, r_{w}}$. Then,

$$
\begin{aligned}
\operatorname{Pr}[\mathrm{I}<R] \sim & {\left[\frac{2^{m R}-1}{\mathrm{SNR}}\right]^{m} \times \lambda_{s, d(s)} \times \sum_{\mathcal{D}(s)}\left[\frac{1}{1-P_{w}}\right]^{m-|\mathcal{D}(s)|-1} } \\
& \times \prod_{r \in \mathcal{D}(s)} \lambda_{r, d(s)} \times \prod_{r \notin \mathcal{D}(s)} \bar{\lambda}_{s, r} \times \frac{1}{(|\mathcal{D}(s)|+1) !}, \text { where } 0 \leq P_{w}<1 .
\end{aligned}
$$

Proof: See appendix A.

\section{Analysis}

A few interesting properties are worth pointing out in (5.8). First, note the term $1 /(1-$ $\left.P_{w}\right)$ in each of the summations. As more power is dedicated to the non-fading mode these terms will tend towards infinity. This matches the intuition that as more power at $s$ is dedicated to the non-fading mode, less will be available for $s$ to transmit to all other relays via the wireless fading link.

Note, that $P_{w}=1$ is a singularity, and as $P_{w} \rightarrow 1$ the outage probability tends toward infinity; the approximation breaks down. (5.4) and (5.8) are valid at high wireless SNR. As $P_{w} \rightarrow 1$, the wireless SNR $\rightarrow 0$ (linear), the range where 5.8 is invalid. In addition, probabilities are values less than one, however as $P_{w} \rightarrow 1$, (5.8) will produce values greater than one. These values are obviously out of the range where the approximation is accurate.

When performing the substitution for the values of $\bar{\lambda}$ notice the scalar factor $\left(2^{m \hat{R}}-\right.$ 1)/(2mR -1$)$ before $\lambda_{s, r_{w}}$. A smaller value of $\lambda_{s, r_{w}}$ corresponds to a better channel. The term $2^{m \hat{R}}-1$ will always be less than $2^{m R}-1$, as long as the non-fading mode provides some non-zero rate. Essentially, the scalar factor lowers the value of $\lambda_{s, r_{w}}$. Intuitively, we can think of the non-fading mode improving the perceived quality of the fading channel $\lambda_{s, r_{w}}$.

\section{Numerical Results}

Figure 5.6 plots simulated outage versus (5.8). In the plot, $R=1$, all $\lambda=1$ except $\lambda_{s, r_{w}}=10$, and $\frac{B_{w}}{B_{w l}}=1$. As the figure shows, the approximation is most accurate at 


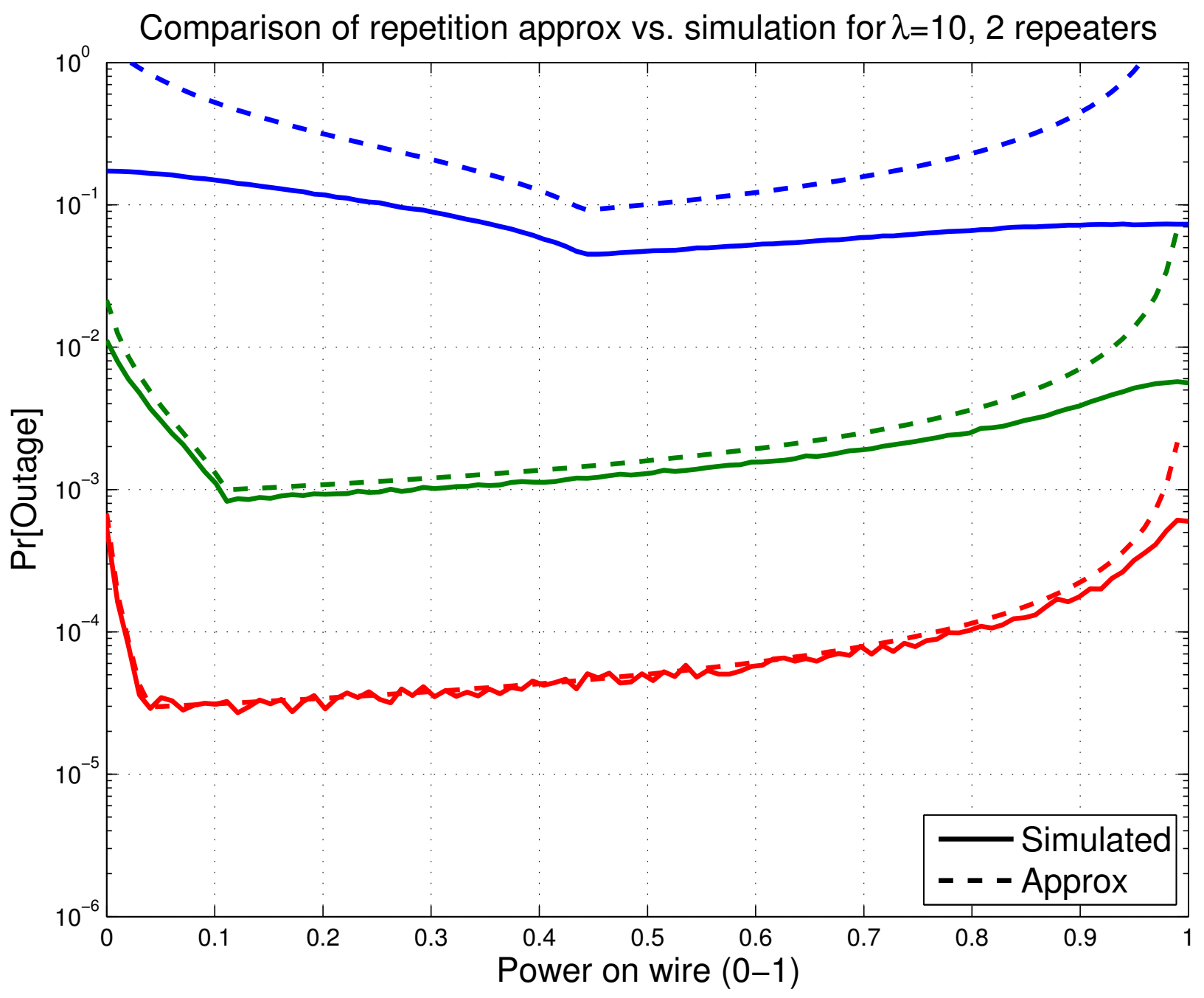

Figure 5.6: Approximation versus simulation results for a repetition scheme with $\mathrm{m}=3$, $\lambda_{s, r_{w}}=10$ all other $\lambda=1$ and $R=1$ for 12,18 and $23 \mathrm{~dB}$

high-SNR in the lower $P_{w}$ regime.

Figure 5.6 demonstrates the tradeoff between dividing power between the fading and non-fading modes. In the extreme, as $P_{w}$ approaches one, all power at $s$ is dedicated to $r_{w}$ and no other relay will be able to decode as no power is dedicated to the wireless fading mode. As the simulated results show, outage does not go to $100 \%$ since even in the extreme $r_{w}$ can decode and retransmit the message.

In figure 5.7, the parameters are the same as figure 5.6, but with $h=0.41$. With a smaller value for $h$, more power must be dedicated to the non-fading mode to guarantee the target rate $R$ is achieved. Indeed, the lower two curves appear to be elongated to the right, with 


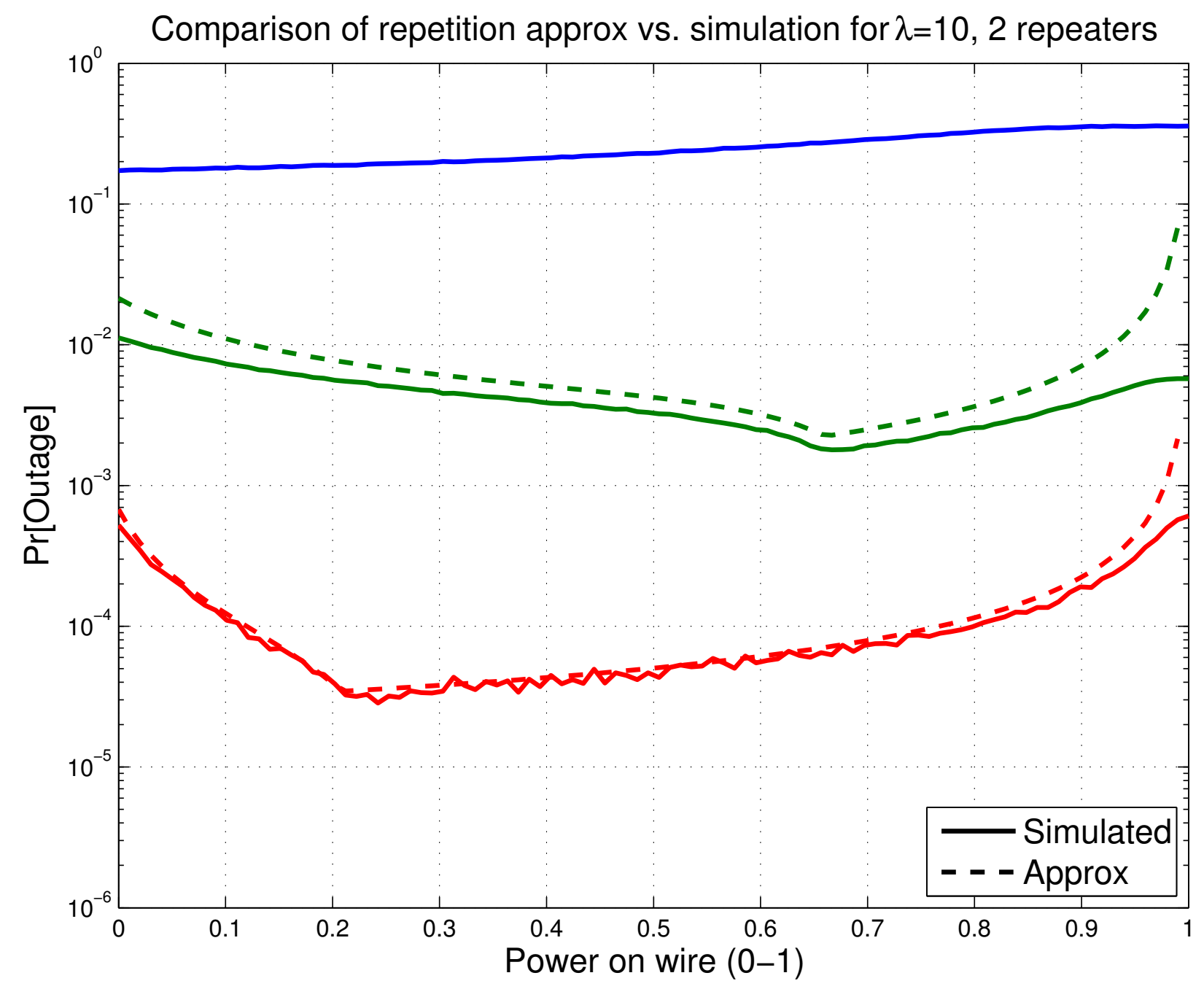

Figure 5.7: Same parameters as in figure 5.6, but with $h=0.41$

the optimal allocation again appearing to be where the non-fading mode achieves target rate $R$. The $18 \mathrm{~dB}$ curve in figure 5.7 demonstrates slightly poorer performance at the optimal point along the curve when compared to figure 5.6. Intuitively, as more power is dedicated to the non-fading mode, less power is available to the fading mode for all other receivers. Thus, for a larger $P_{w}$ to achieve the target rate $R$ we expect poorer outage performance. 


\subsection{Non-fading Mode from Relay-to-Destination}

Unfortunately, for the repetition scheme, we were unable to develop approximations for an additional non-fading mode between a relay $r_{w}$ and the destination $d(s)$. However, in this section we provide numerical results from simulations and comment on the system's behavior.

\section{Numerical Results}

In figure 5.8 below, we've presented three plots. In each plot, $R=1, h=1, \frac{B_{w}}{B_{w l}}=1$. $\lambda=1$ for all $r \neq r_{w}$. In 5.8(a) $\lambda_{r_{w}, d(s)}=1$, in 5.8(b) $\lambda_{r_{w}, d(s)}=10$ and in 5.8(c) $\lambda_{r_{d}, d(s)}=100$. These plots show some different characteristics than the plot for a source-relay non-fading mode.

Contrasted with the plot in figure 5.6, we can see this plot does not suffer increased outage in the higher $P_{w}$ regime. This corresponds to the relay dedicating all of it's transmission power to the non-fading mode. Assuming the non-fading mode available to $r_{w}$ can achieve the target rate $R$ there is no penalty since no other nodes are listening to the transmission of $r_{w}$. This is not the case for a source-relay non-fading mode. In that scenario, $s$ is the origin of transmissions, and power sacrificed to the non-fading mode is power lost to the relays attempting to overhear the fading transmission of $s$.

In addition, similar to the source-relay configuration, the optimal power allocation appears to be in the same regime. The optimal strategy appears to be providing enough power for the non-fading mode to achieve the target rate $R$. However, precision in adjusting $P_{w}$ does not appear to be quite as important, and the optimal outage can be achieved over a wide range of $P_{w}$.

\subsection{Summary}

In this chapter we examined a protocol for cooperative communication using repetition. We presented previous work on the topic, and proposed introducing a non-fading mode between both the source and a relay, and a relay and the destination, separately. We pro- 
vided an outage approximation for a single additional source-relay non-fading mode. The approximation is a generalization of the approximation presented in [16], (12).

We were unable to develop an outage approximation for an additional relay-destination non-fading mode. However, we did compare simulation results to the source-relay configuration. In the relay-destination non-fading mode case the optimal outage appears to occur over a wide range of power allocations in contrast to the apparent precision necessary in the source-relay configuration. In both cases, optimal outage appears to be achieved when the exact amount of power required by the non-fading mode to decode is provided, but no more. 


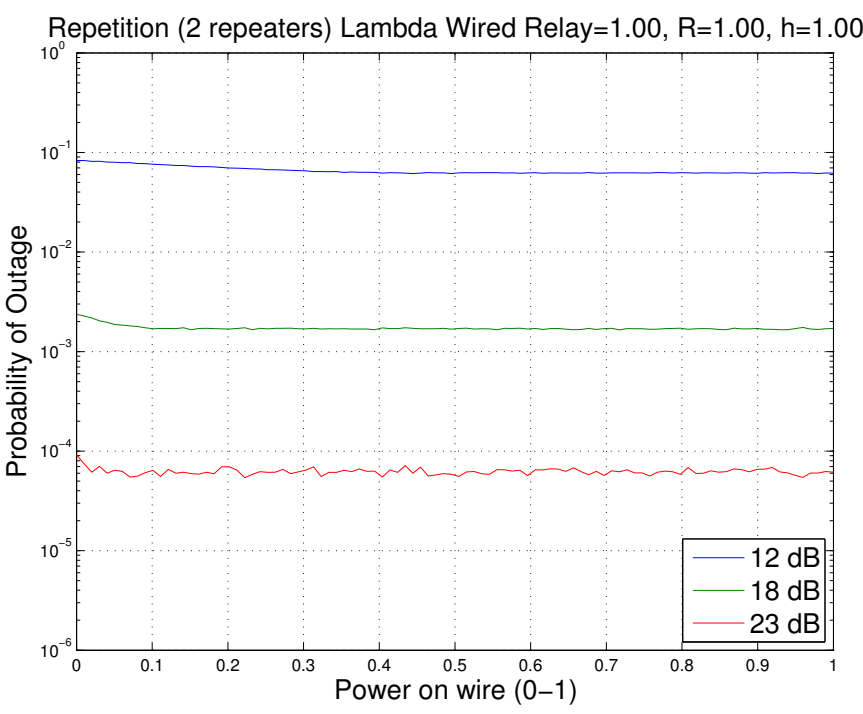

(a) $\lambda_{r_{w}, d(s)}=1$

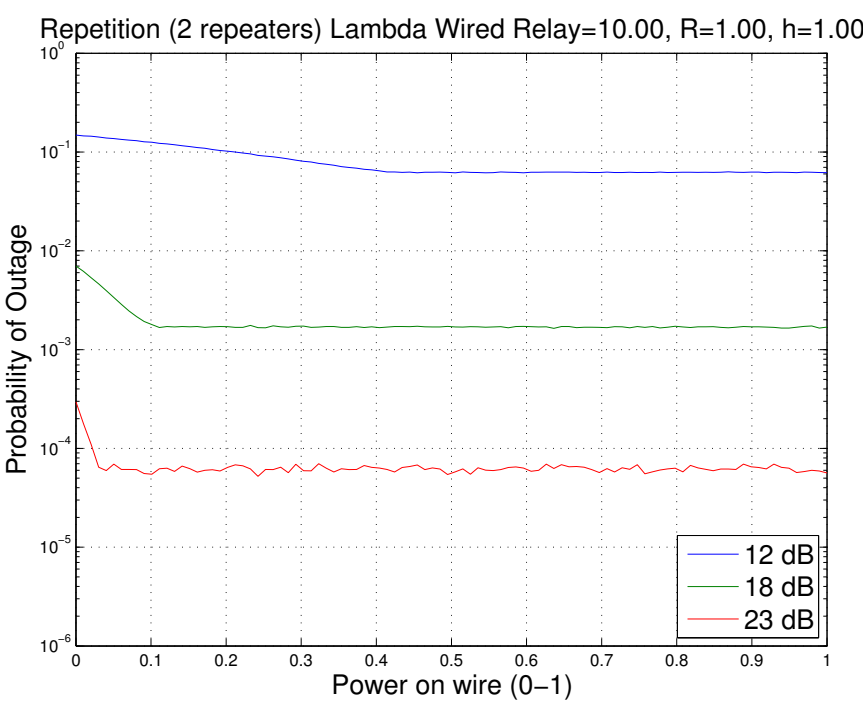

(b) $\lambda_{r_{w}, d(s)}=10$

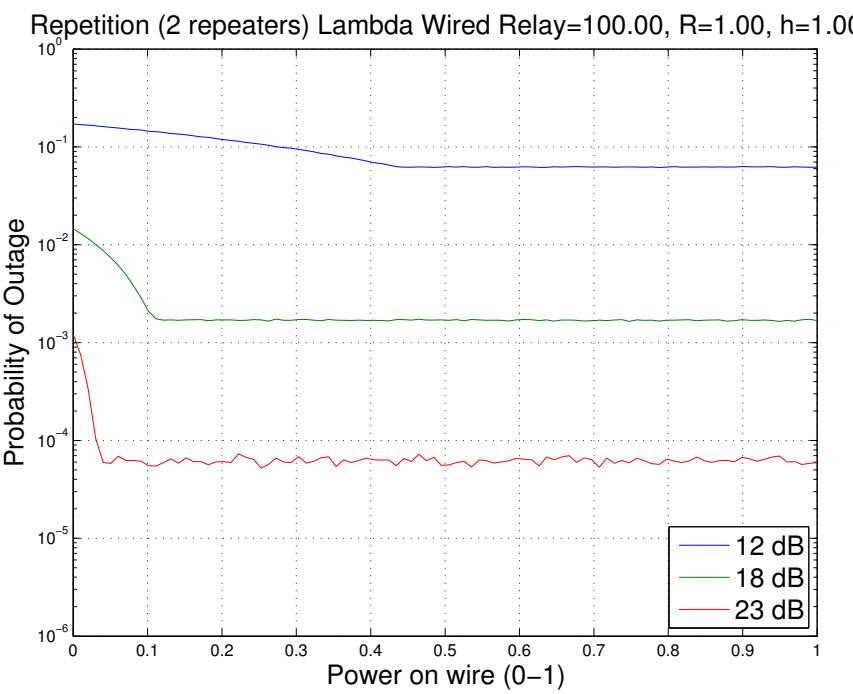

(c) $\lambda_{r_{w}, d(s)}=100$

Figure 5.8: Simulated outage for a two-relay network with a non-fading mode between $r_{w}$ and $d(s)$. Figure (a) represents a network with $\lambda_{r_{w}, d(s)}=1,(\mathrm{~b})$ are results for $\lambda_{r_{w}, d(s)}=10$ and (c) for $\lambda_{r_{w}, d(s)}=100$. 


\section{Chapter 6}

\section{Multimodal Cooperation Using Distributed Space-Time Coding}

In this chapter, we maintain the system network structure developed in the previous chapter. The network still consists of $m$ transmitters with a source $s$ and destination $d(s)$. Again, we will analyze the addition of a non-fading mode between both a source and relay, and a relay and destination. However, the MAC presented in this chapter does not use a repetition scheme.

In this chapter, $s$ will transmit a message intended for $d(s)$ which the relays will overhear. However, rather than taking turns in orthogonal time-slots, relays will transmit simultaneously using a distributed space-time code (DSTC). Since there are only two phases of transmission rather than $m$, adjustments will be made to the system model.

Much of this chapter builds on ideas and strategies developed in the previous chapter. The reader is strongly encouraged to read the previous chapter in its entirety before beginning this chapter.

\subsection{Medium Access Control}

As shown in figure 6.1, in the first time-slot the source transmits a message intended for $d(s)$. In the second time-slot, relays that overhear the message and successfully decode, denoted $\mathcal{D}(s)$, relay the message simultaneously to the destination $d(s)$. As previously men- 
tioned, changes must be introduced into the system model to account for the augmentation of the degrees-of-freedom used.

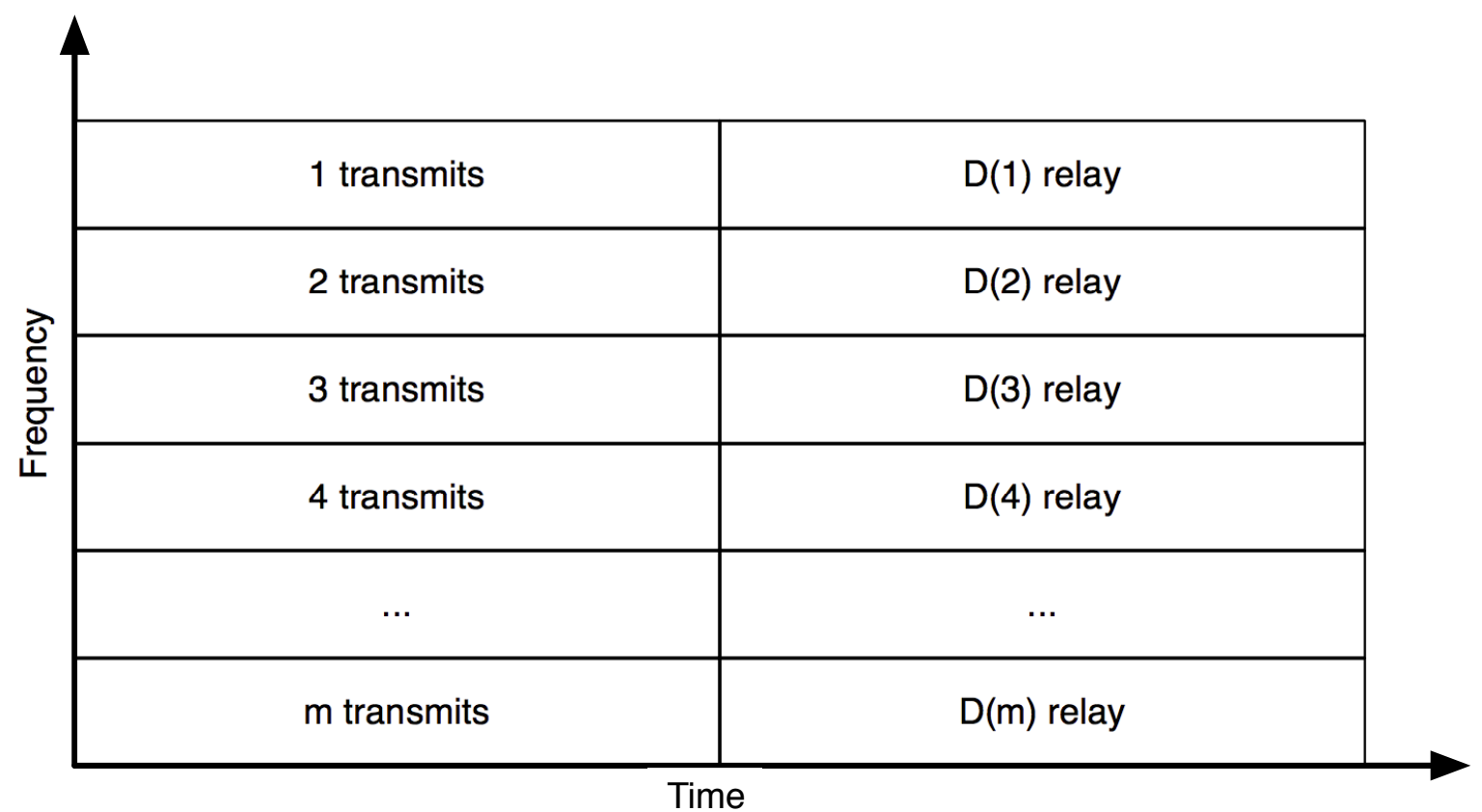

Figure 6.1: Medium access control for space-time cooperation

\subsection{System Model}

Since each transmitter now uses $1 / 2$ the degrees-of-freedom for transmission rather than the fraction $1 / m$, the transmit SNR must be modified. For the space-time case, we normalize the transmit SNR

$$
\frac{2}{m} \mathrm{SNR}=\frac{2}{m} \times \frac{m P}{N_{0} W}=\frac{2 P}{N_{0} W}
$$

This normalized SNR was developed in [16] to allow for fair comparisons between repetition and space-time schemes. We maintain this normalization.

The necessary mutual information for a relay to participate in the decoding set also must be modified to match the new SNR normalization. In this chapter, the realized mutual 
information between $s$ and relay $r$ is

$$
\frac{1}{2} \log \left(1+\frac{2}{m} \operatorname{SNR}\left|a_{s, r}\right|^{2}\right)
$$

Thus, for a relay to participate in the decoding set,

$$
\begin{aligned}
\operatorname{Pr}[r \in \mathcal{D}(s)] & =\operatorname{Pr}\left[\frac{1}{2} \log \left(1+\frac{2}{m} \operatorname{SNR}\left|a_{s, r}\right|^{2}\right)>R\right] \\
& =\operatorname{Pr}\left[\left|a_{s, r}\right|^{2}>\left(\frac{2^{2 R}-1}{2 \mathrm{SNR} / m}\right)\right] .
\end{aligned}
$$

As in the previous chapter, $\left|a_{s, r}\right|^{2}$ is an exponential random variable distributed with parameter $\lambda_{s, r}$. Thus, 6.3) is simply the CDF of a random variable, which is the same as the preceding chapter.

\subsection{Outage Probability Without Additional Modes}

It was shown in [16], for a cooperative network of $m$ transmitters using the space-time protocol described in section 6.2 , the following outage approximation is valid at high-SNR,

$$
\begin{aligned}
\operatorname{Pr}[\mathrm{I}<R] \sim & {\left[\frac{2^{2 R}-1}{2 \mathrm{SNR} / m}\right]^{m} \times \sum_{\mathcal{D}(s)} \lambda_{s, d(s)} } \\
& \times \prod_{r \notin \mathcal{D}(s)} \lambda_{r, d(s)} \prod_{r \notin \mathcal{D}(s)} \lambda_{s, r} \times A_{|\mathcal{D}(s)|}\left(2^{2 R}-1\right) .
\end{aligned}
$$

Figure 6.2 plots (6.4) for several values of $m, R=1$ and $\lambda=1$ for all channels.

\subsection{Outage Approximation for an Additional Source- Relay Non-Fading Mode}

We now analyze the network when a single additional non-fading mode is placed between the source $s$, and a relay $r_{w}$.

Proposition 2: Let $\bar{\lambda}_{s, r}=\lambda_{s, r}$ for all $r \neq r_{w}$. Let $\bar{\lambda}_{s, r_{w}}=\left(2^{2 \hat{R}}-1\right) /\left(2^{2 R}-1\right) \lambda_{s, r_{w}}$. Then,

$$
\begin{aligned}
\operatorname{Pr}[\mathrm{I}<R] \sim & {\left[\frac{2^{2 R}-1}{2 \mathrm{SNR} / m}\right]^{m} \times \lambda_{s, d(s)} \times \sum_{\mathcal{D}(s)}\left[\frac{1}{1-P_{w}}\right]^{m-|\mathcal{D}(s)|-1} } \\
& \times \prod_{r \in \mathcal{D}(s)} \lambda_{r, d(s)} \times \prod_{r \notin \mathcal{D}(s)} \bar{\lambda}_{s, r} \times A_{|\mathcal{D}(s)|}\left(2^{2 R}-1\right) .
\end{aligned}
$$

Proof: See appendix B. 


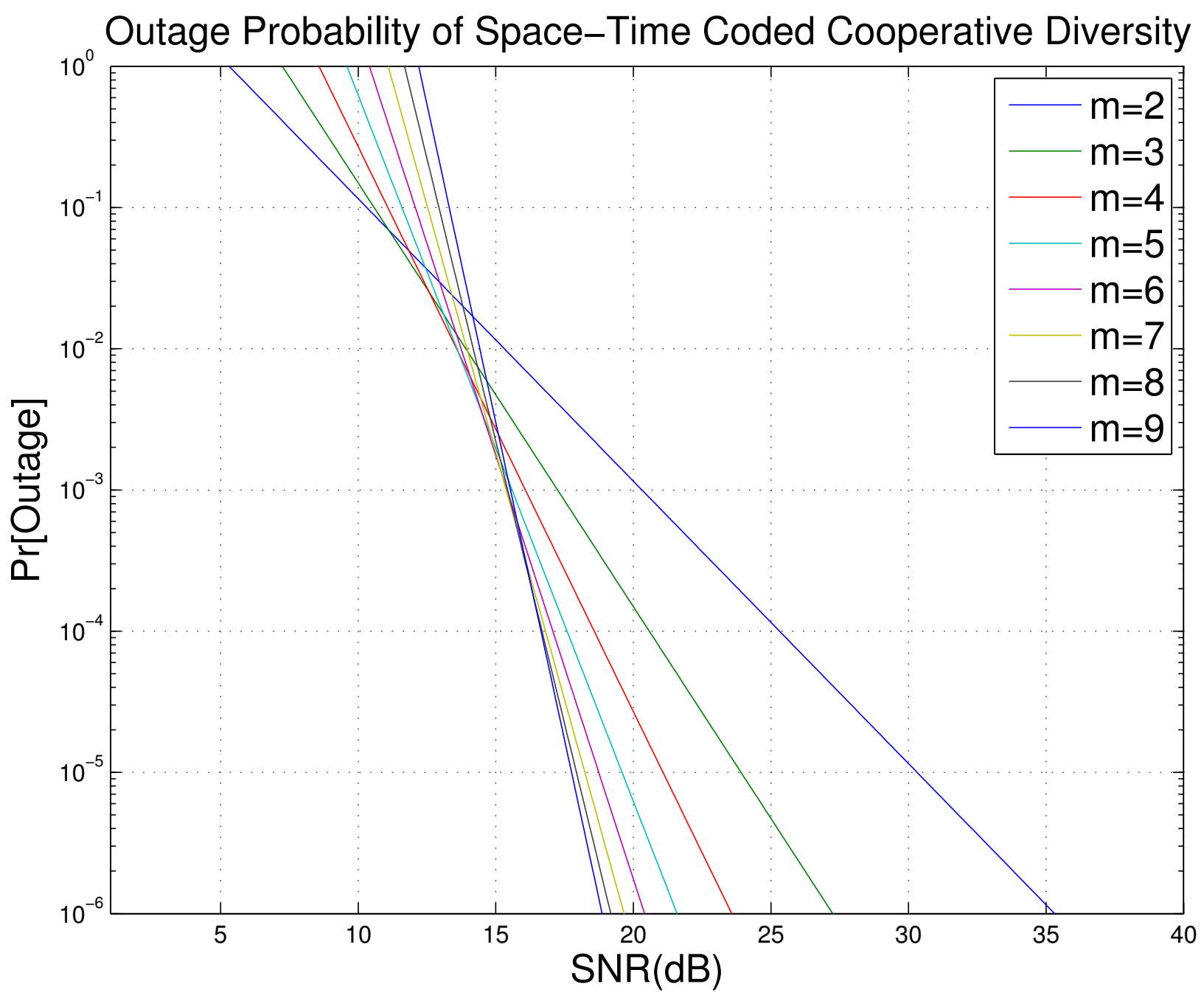

Figure 6.2: Space-time outage probability for a wireless network for several values of $m$, $R=1$, and $\lambda=1$ for all channels.

\section{Analysis}

Examining (7.13), it is the same form as [16], equation (19) with the addition of a scale factor dependent on the power dedicated to the non-fading mode in each term of the summation. As long as $P_{w}<1$, we can see the system maintains full diversity. This is shown by the exponent of the inverse SNR term in the equation. It can also be seen that as $P_{w}$ increases, the term $\left(1 /\left(1-P_{w}\right)\right)^{m-|\mathcal{D}(s)|-1}$ will tend toward infinity. Note that, as in the previous chapter, the approximation reaches a singularity as $P_{w} \rightarrow 1$ and any approximation greater than one is invalid. Additionally, (7.13) is a generalization of (6.3), which can be 
seen by substituting $P_{w}=0$.

This is in competition with the term $\hat{\lambda}_{s, r_{w}}=\left(2^{2 \hat{R}}-1\right) /\left(2^{2 R}-1\right) \lambda_{s, r_{w}}$. Again, since $\left(2^{2 \hat{R}}-1\right) \leq\left(2^{2 R}-1\right), \bar{\lambda}_{s, r_{w}}$ is always smaller than $\lambda_{s, r}$, indicating a better channel quality. The numerical results in the next section will help illustrate this tradeoff.

\section{Numerical Results}

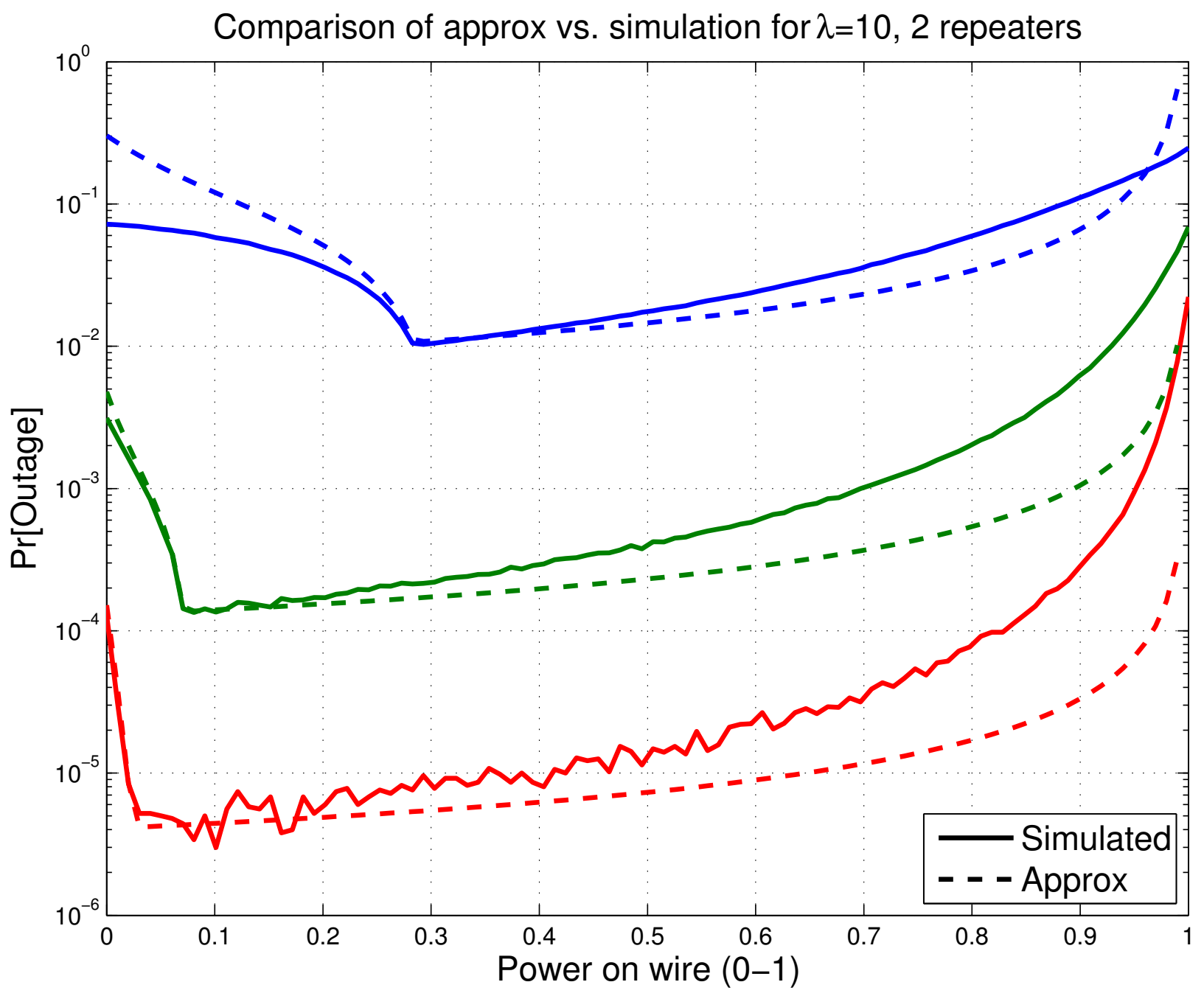

Figure 6.3: Approximation versus simulation results for a space-time scheme with an additional non-fading mode between $s$ and $r_{w}, m=3, \lambda_{s, r_{w}}=10$ all other $\lambda=1$ and $R=1$ for 12,18 and $23 \mathrm{~dB}$ when $P_{w}=0$

In figure 6.3, we plot simulated outage results versus the approximation in (7.13). We plot the outage across a variety of SNRs, holding $R=1, h=1$, and $\frac{B_{w}}{B_{w l}}=1$. All $\lambda_{r, d(s)}=$ 
$1, \bar{\lambda}_{s, r}=1$, except $\bar{\lambda}_{s, r_{w}}=\left(2^{2 \hat{R}}-1\right) /\left(2^{2 R}-1\right) \lambda_{s, r_{w}}$, where

$\hat{R}=R-\frac{B_{w}}{2 B_{w l}} \log \left(1+\frac{P_{w} B_{w l}}{B_{w}} \frac{2}{m} \operatorname{SNR}|h|^{2}\right)$.

As in the previous chapter, the tradeoff between $s$ diverting power to the non-fading mode instead of the fading mode can be seen. As we've seen before, in the extreme where $P_{w} \sim 1$ the outage approximation tends to infinity, as we might expect. The approximation is particularly good around the area where power dedicated to the non-fading achieves the target rate $R$.

Figure 6.4 plots outage for a network with no additional fading modes versus a single additional fading mode between $s$ and a single relay. $P_{w}$ is adjusted on the non-fading mode such that the target rate $R=1$ is achieved, if possible. All $\lambda=1, \frac{B_{w}}{B_{w l}}=1$ and $h=1$.

\subsection{Outage Approximation for an Additional Relay- Destination Non-Fading Mode}

In this section we analyze the outage when a single non-fading mode is present between a relay $r_{w}$ and destination $d(s)$. In this scenario, $r_{w}$ must divide its finite transmit power between the fading and non-fading mode. Again, the percentage of power dedicated to the non-fading mode will be denoted $P_{w}, P_{w} \in[0,1]$. We assume the non-fading mode has bandwidth $B_{w}$ and complex frequency gain $h$ that we assume is constant over the bandwidth of interest.

In the previous section, we analyzed the impact a non-fading mode from $s$ to $r_{w}$ would have on the probability of particular decoding sets $\mathcal{D}(s)$. Expressions for the second-phase of transmission, relays transmitting simultaneously, were employed without any further modification. With a non-fading between $r_{w}$ and $d(s)$ the situation is mirrored. The non-fading mode will not affect the first-phase of communication, $\operatorname{Pr}[\mathcal{D}(s)]$, but new expressions for the second-phase of communication $\operatorname{Pr}[\mathbf{I}<R \mid \mathcal{D}(s)]$ must be developed. 


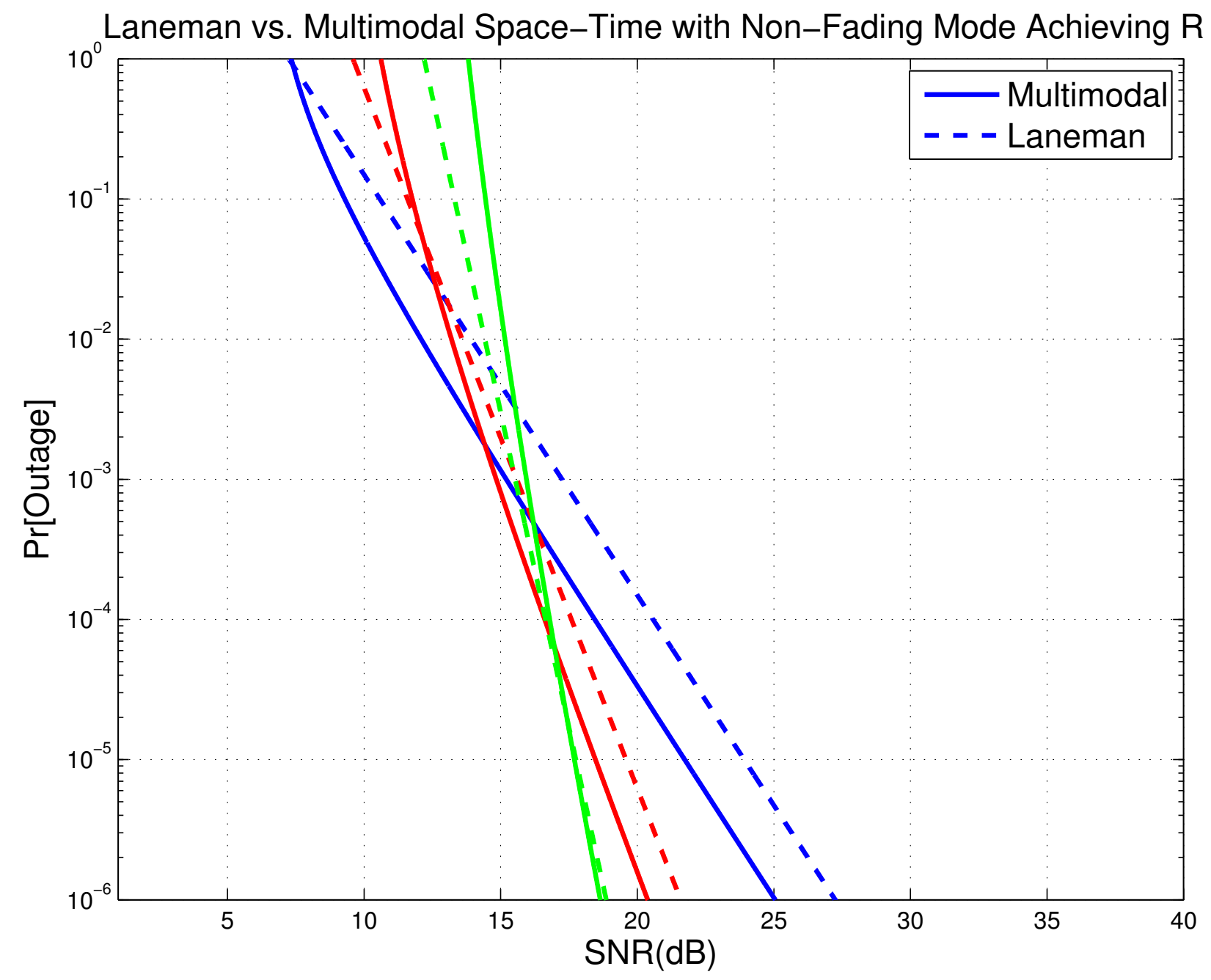

Figure 6.4: Comparison of networks with no additional fading modes versus a single nonfading mode between source and relay. Plots are for $m=3$ (blue), $m=5$ (red) and $m=9$ (green). $\lambda=1$ for all channels. $P_{w}$ is configured to allow the non-fading mode to achieve target rate $R=1$.

Proposition 3: Let $\bar{\lambda}_{r, d(s)}=\lambda_{r, d(s)}, r \neq r_{w}$. Let $\bar{\lambda}_{r_{w}, d(s)}=\frac{\lambda_{r_{w}, d(s)}}{\left(1-P_{w}\right)}$. Then,

$$
\begin{aligned}
\operatorname{Pr}[\mathrm{I}<R] \sim & {\left[\frac{2^{2 R}-1}{2 \mathrm{SNR} / m}\right]^{m} \times \lambda_{s, d(s)} } \\
\times & \left\{\sum_{\substack{\mathcal{D}(s): \\
r_{w} \in \mathcal{D}(s)}}\left[\frac{2^{2 \hat{R}}-1}{2^{2 R}-1}\right]^{|\mathcal{D}(s)|+1} \times \prod_{r \in \mathcal{D}(s)} \bar{\lambda}_{r, d(s)} \times \prod_{r \notin \mathcal{D}(s)} \lambda_{s, r} \times A_{|\mathcal{D}(s)|}\left(2^{2 \hat{R}}-1\right)\right. \\
& \left.+\sum_{\substack{\mathcal{D}(s): \\
r_{w} \notin \mathcal{D}(s)}} \prod_{r \in \mathcal{D}(s)} \bar{\lambda}_{r, d(s)} \times \prod_{r \notin \mathcal{D}(s)} \lambda_{s, r} \times A_{|\mathcal{D}(s)|}\left(2^{2 R}-1\right)\right\} .
\end{aligned}
$$

Proof: See appendix C. 


\section{Analysis}

(6.6) closely resembles the form of [16], (22). The equation remains partitioned over decoding sets including and excluding $r_{w}$. When $r_{w} \notin \mathcal{D}(s)$, the second summation, the form is the same. When $r_{w} \in \mathcal{D}(s)$ the form is similar, with a scaled version of $\lambda_{r_{w}, d(s)}$. We can see that $\left[\frac{2^{2 \hat{R}}-1}{2^{2 R}-1}\right]^{|\mathcal{D}(s)|+1}$ and $\bar{\lambda}_{r_{w}, d(s)}$ are at odds. As $P_{w}$ increases, the former decreases while the latter increases. This matches intuitively with our understanding. As more power is directed toward the non-fading mode the fading channel quality suffers to the benefit of improved SNR on the non-fading mode.

\section{Numerical Results}

Figure 6.5 shows similar behavior to the simulation curves shown in the repetition chapter for a non-fading mode between a relay $r$ and destination $d(s)$. Again, the optimal power allocation appears to be when sufficient $P_{w}$ is provided for the non-fading mode to achieve the target rate $R$. Note that the penalty for exceeding $P_{w}$ to achieve $R$ is not present in this plot. In the second stage, the destination $d(s)$ is the only node intended for reception, so if the rate can be achieved there is no penalty for directing power away from the fading mode.

\subsection{A Note on Path Loss}

Up to this point, we've only mentioned path loss in passing. Path loss is the degradation of message energy as it passes through the wireless channel. In [9], a simplified model for path loss was presented

$$
P_{r}=P_{t} K\left[\frac{d_{0}}{d}\right]^{\gamma}
$$

where $P_{r}$ is the power at the receiver and $P_{t}$ is the transmit power. $K, d_{0}$ and $\gamma$ are constants used to shape the path loss to a specific environment, and $d$ is the distance between transmitter and receiver.

When including path-loss we would model the received message

$$
y=\sqrt{K\left[\frac{d_{0}}{d}\right]^{\gamma}} a x+z,
$$




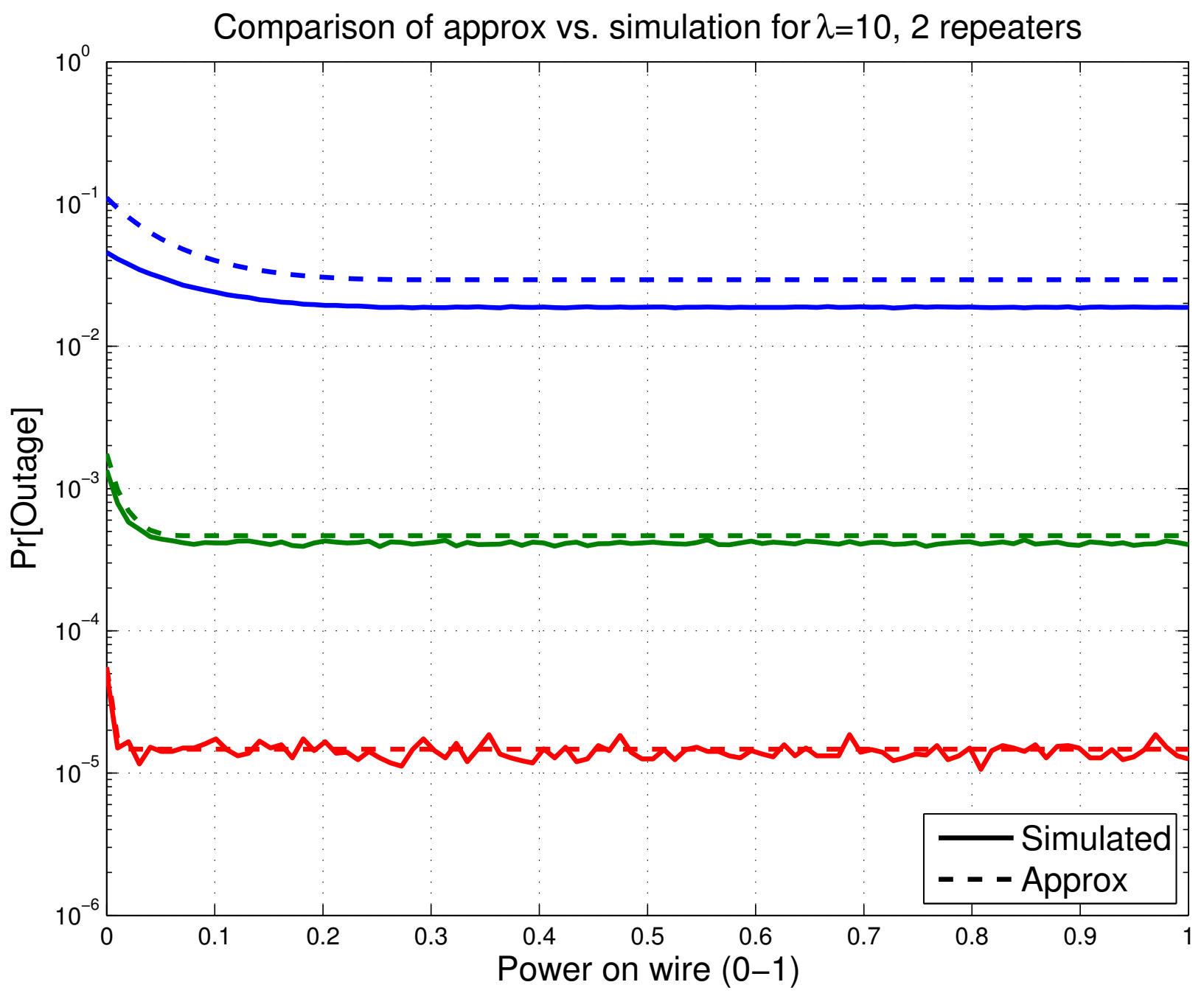

Figure 6.5: Approximation versus simulation results for a space-time scheme with an additional non-fading mode between $r_{w}$ and $d(s), m=3, \lambda_{r_{w}, d(s)}=10$ all other $\lambda=1$ and $R=1$ for 12,18 and $23 \mathrm{~dB}$ when $P_{w}=0$

where $a$ is the fading coefficient, $x$ is the source message and $z$ the additive noise. The receive SNR will then be

$$
|a|^{2} K\left[\frac{d_{0}}{d}\right]^{\gamma} \text { SNR }
$$

Note that the path loss is deterministic, and that $|a|^{2}$ is a random variable. Specifically, $|a|^{2}$ is an exponential random variable with parameter $\lambda$. 
Consider a new random variable, $Y=\left|a^{2}\right| K\left[\frac{d_{0}}{d}\right]^{\gamma}$. Then

$$
\begin{aligned}
\operatorname{Pr}[Y<y] & =\operatorname{Pr}\left[|a|^{2} K\left[\frac{d_{0}}{d}\right]^{\gamma}<y\right] \\
& =\operatorname{Pr}\left[|a|^{2}<\frac{y}{K}\left[\frac{d}{d_{0}}\right]^{\gamma}\right] \\
& =1-\exp \left(-\frac{\lambda}{K}\left[\frac{d}{d_{0}}\right]^{\gamma} y\right)
\end{aligned}
$$

Substituting $\bar{\lambda}=\frac{\lambda}{K}\left[\frac{d}{d_{0}}\right]^{\gamma}$ back into the previous equation yields

$$
\operatorname{Pr}[Y<y]=1-\exp (-\bar{\lambda} y)
$$

6.11) is still the CDF for an exponential random variable. This means that while path loss wasn't specifically pointed out in our system model, we can include it by manipulating the values of $\lambda$ for each wireless channel without any additional changes to our model. This applies to both the repetition and space-time schemes.

\subsection{Summary}

In this chapter we presented an existing protocol for a space-time cooperative network. We introduced an additional non-fading mode between the source and a relay in the network. We also presented a system with a single additional non-fading mode placed between a relay and the destination. We've shown that both of these systems maintain full diversity, assuming $P_{w}<1$. The additional non-fading mode can allow a designer to achieve better outage performance if placed between nodes that have a poor wireless link.

As in the repetition case, if we can precisely control power between fading and nonfading modes, a source-relay non-fading mode appears to provide a slightly improved outage performance than a relay-destination non-fading mode. We've also pointed out that pathloss, while not explicitly pointed out, can be integrated into our model. 


\section{Chapter 7}

\section{Extension to Multiple Modes}

\subsection{Adding Multiple Modes}

We examine extending our model to multiple modes between source $s$ and several relaying nodes. In previous sections we denoted the single relay connected to the non-fading mode $r_{w}$. We now introduce new notation to denote connections to multiple non-fading modes. Assume a subset $W$ of relays are connected to the source $s$. Then $W \subseteq\{M-s\}$ and $1 \leq|W| \leq m-1$.

Each of these non-fading links have independent properties; frequency response $h$ and normalized bandwidth $B_{w} / B_{w l}$. For every $r \in W, h_{r}$ will denote the frequency response of the non-fading mode between $s$ and $r$. Dealing with the bandwidth notation will be a bit cumbersome, so we introduce new notation, $B=B_{w} / B_{w l}$. Therefore, the normalized bandwidth for the non-fading link between $s$ and $r$ is denoted $B_{r}, \forall r \in W$.

Once again, $s$ must divide transmission power between the fading mode, and in this extended case, multiple non-fading modes. $P_{r}$ will denote the percentage of overall power used for transmission over the non-fading mode between $s$ and $r, \forall r \in W$. We shall denote the total amount of power dedicated to all non-fading modes $P_{w}$, defined as

$$
P_{w}=\sum_{r \in W} P_{r}
$$

Note that the sum of all power dedicated to all non-fading modes must not exceed the total power constraint for $s, P_{w} \leq 1$. In essence, the meaning of $P_{w}$, and $\left(1-P_{w}\right)$ remains the 
same; the total power dedicated to non-fading transmission and the remaining power left for transmission over the fading mode, respectively. In the extreme where $P_{w}=1$, all power will be dedicated to the non-fading modes, and no power will remain available to the fading mode.

\subsection{Multiple Source-Relay Modes for Repetition Scheme}

As mentioned in chapter 5, an additional non-fading mode between source and relays impacts only $\operatorname{Pr}[\mathcal{D}(s)]$. This statement also holds for multiple non-fading modes between source and relays. For $r \in W$, the instantaneous information between $s$ and $r$ is

$$
\mathrm{I}_{\mathrm{s}, \mathrm{r}}=\frac{1}{m} \log \left(1+\left(1-P_{w}\right) \operatorname{SNR}\left|a_{s, r}\right|^{2}\right)+\frac{B_{r}}{m} \log \left(1+\operatorname{SNR} \frac{\left|h_{r}\right|^{2} P_{r}}{B_{r}}\right) .
$$

This equation is very similar to $\mathrm{A}-8 \mathrm{~B}$. In general, it is the exact same equation with the specifics of the non-fading mode between $s$ and $r$ inserted. (7.2) holds for all $r \in W$. The probability of $r \in W$ participating in the decoding set is then

$$
\begin{aligned}
\operatorname{Pr}[r \in \mathcal{D}(s)]= & \operatorname{Pr}\left[\mathrm{I}_{\mathrm{s}, \mathrm{r}}>R\right] \\
= & \operatorname{Pr}\left[\frac{1}{m} \log \left(1+\left(1-P_{w}\right) \operatorname{SNR}\left|a_{s, r_{w}}\right|^{2}\right)\right. \\
& \left.+\frac{B_{r}}{m} \log \left(1+\operatorname{SNR} \frac{|h|^{2} P_{r}}{B_{r}}\right)>R\right] \\
= & \operatorname{Pr}\left[\frac{1}{m} \log \left(1+\left(1-P_{w}\right) \operatorname{SNR}\left|a_{s, r_{w}}\right|^{2}\right)\right. \\
& \left.>R-\frac{B_{r}}{m} \log \left(1+\operatorname{SNR} \frac{|h|^{2} P_{r}}{B_{r}}\right)\right] .
\end{aligned}
$$

Again, the term on the right-hand side of the inequality is entirely deterministic. We perform the substitution $\hat{R}_{r}=R-\frac{B_{r}}{m} \log \left(1+\operatorname{SNR} \frac{|h|^{2} P_{r}}{B_{r}}\right)$. 7.3p then becomes

$$
\operatorname{Pr}\left[r_{w} \in \mathcal{D}(s)\right]=\operatorname{Pr}\left[\left|a_{s, r}\right|^{2}>\frac{2^{m \hat{R}_{r}}-1}{\left(1-P_{w}\right) \operatorname{SNR}}\right] .
$$

(7.3) is of the same form as $\mathrm{A}-10 \mathrm{D}$. This implies we can use the same strategy for developing approximations. Once again leveraging the Taylor series we develop the approximations for the relays attached to non-fading modes

$$
\operatorname{Pr}[r \in \mathcal{D}(s)] \sim 1-\lambda_{s, r} \frac{2^{m \hat{R}_{r}}-1}{\left(1-P_{w}\right) \mathrm{SNR}}
$$


and

$$
\operatorname{Pr}[r \notin \mathcal{D}(s)] \sim \lambda_{s, r} \frac{2^{m \hat{R}_{r}}-1}{\left(1-P_{w}\right) \mathrm{SNR}}
$$

For manipulation in later steps, we multiply both equations by $\left(2^{m R}-1\right) /\left(2^{m R}-1\right)$.

$$
\operatorname{Pr}[r \in \mathcal{D}(s)] \sim 1-\lambda_{s, r} \frac{2^{m \hat{R}_{r}}-1}{\left(1-P_{w}\right) \operatorname{SNR}} \times \frac{2^{m R}-1}{2^{m R}-1}
$$

and

$$
\operatorname{Pr}[r \notin \mathcal{D}(s)] \sim \lambda_{s, r} \frac{2^{m \hat{R}_{r}}-1}{\left(1-P_{w}\right) \mathrm{SNR}} \times \frac{2^{m R}-1}{2^{m R}-1} .
$$

Now, focusing again on $\operatorname{Pr}[\mathcal{D}(s)]$, since each relay $r$ decodes independently,

$$
\operatorname{Pr}[\mathcal{D}(s)]=\prod_{r \in \mathcal{D}(s)} \operatorname{Pr}[r \in \mathcal{D}(s)] \times \prod_{r \notin \mathcal{D}(s)} \operatorname{Pr}[r \notin \mathcal{D}(s)]
$$

At high-SNR we see that $\operatorname{Pr}[r \in \mathcal{D}(s)]$ goes to unity, and we can ignore this term in $\operatorname{Pr}[\mathcal{D}(s)]$. Now, let's introduce the notation $\bar{\lambda}_{s, r}=\lambda_{s, r}, \forall r \notin W$, and $\bar{\lambda}_{s, r}=\left(2^{m \hat{R}_{r}}-1\right) /\left(2^{m R}-1\right) \lambda_{s, r}, \forall r \in W$. We can then write the approximation in 7.8 as

$$
\operatorname{Pr}[r \notin \mathcal{D}(s)] \sim \bar{\lambda}_{s, r} \frac{2^{m R}-1}{\left(1-P_{w}\right) \operatorname{SNR}}
$$

Now, we approximate $\operatorname{Pr}[\mathcal{D}(s)]$ as

$$
\operatorname{Pr}[\mathcal{D}(s)] \sim\left[\frac{2^{m R}-1}{\left(1-P_{w}\right) \operatorname{SNR}}\right]^{m-|\mathcal{D}(s)|-1} \times \prod_{r \notin \mathcal{D}(s)} \bar{\lambda}_{s, r} .
$$

With 7.10 we can now include the unaltered $\operatorname{Pr}[I<R \mid \mathcal{D}(s)]$ to write the approximation for outage probability with multiple source-relay non-fading links as

$$
\begin{aligned}
\operatorname{Pr}[\mathrm{I}<R] \sim & {\left[\frac{2^{m R}-1}{\mathrm{SNR}}\right]^{m} \times \lambda_{s, d(s)} \times \sum_{\mathcal{D}(s)}\left[\frac{1}{1-P_{w}}\right]^{m-|\mathcal{D}(s)|-1} } \\
& \times \prod_{r \in \mathcal{D}(s)} \lambda_{r, d(s)} \times \prod_{r \notin \mathcal{D}(s)} \bar{\lambda}_{s, r} \times \frac{1}{(|\mathcal{D}(s)|+1) !}
\end{aligned}
$$

which is a generalization of $(5.8)$, with substitutions for $\lambda$ connected the source via a nonfading mode. 


\subsection{Multiple Source-Relay Modes for Space-Time Scheme}

The space-time derivation follows the same mechanics as the repetition case. We achieve the final approximation for multiple non-fading modes between the source and multiple relays

$$
\begin{aligned}
\operatorname{Pr}[\mathrm{I}<R] \sim & {\left[\frac{2^{2 R}-1}{2 \mathrm{SNR} / m}\right]^{m} \times \lambda_{s, d(s)} \times \sum_{\mathcal{D}(s)}\left[\frac{1}{1-P_{w}}\right]^{m-|\mathcal{D}(s)|-1} } \\
& \times \prod_{r \in \mathcal{D}(s)} \lambda_{r, d(s)} \times \prod_{r \notin \mathcal{D}(s)} \bar{\lambda}_{s, r} \times A_{|\mathcal{D}(s)|}\left(2^{2 R}-1\right), \text { where } 0 \leq P_{w}<1(7.1
\end{aligned}
$$

with $\bar{\lambda}_{s, r}=\lambda_{s, r}, \forall r \notin W$, and $\bar{\lambda}_{s, r}=\left(2^{2 \hat{R}_{r}}-1\right) /\left(2^{2 R}-1\right) \lambda_{s, r}, \forall r \in W$. However, in this case, $\hat{R}_{r}=R-\frac{B_{r}}{2} \log \left(1+\frac{P_{r}}{B_{r}} \frac{2}{m} \operatorname{SNR}|h|^{2}\right)$.

Figure 7.1 plots outage for a two-relay network with non-fading modes between $s$ and both relays. The characteristics of the modes are both $B_{r}=1$ and $h_{r}=1$. $P_{r}$ is incremented from 0 to 0.5 for both modes equally. Total power dedicated to the non-fading modes is plotted along the $x$-axis, such that all transmit power is dedicated to the non-fading modes when each is given half of the overall power. $R=1, \lambda s, r=10$ for both relays and all other $\lambda=1$.

The curves in figure 7.1 exhibits behavior similar to those shown for a single non-fading mode between source and relay, such as figure 6.3. Notice, however, that after the optimal point along the curves the approximation breaks down. The approximation does not capture loss of the $s-d(s)$ diversity path as $P_{w}$ increases. 


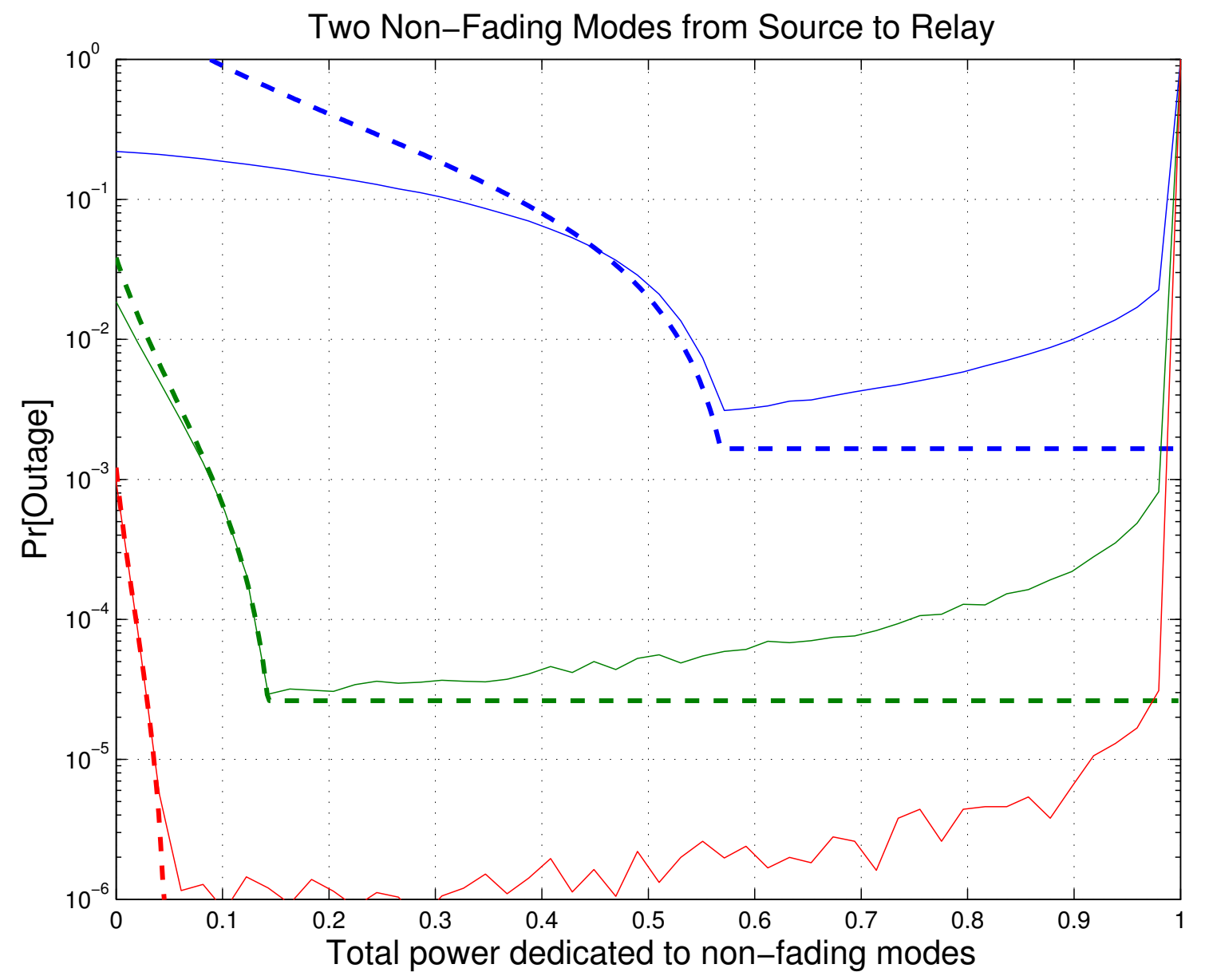

Figure 7.1: Approximation versus simulation results for a space-time scheme for a two-relay network with non-fading modes to both relays. $\lambda_{s, r}=10$ for both relays. All other $\lambda=1$ and $R=1$ for 12,18 and $23 \mathrm{~dB}$ when $P_{w}=0$ 


\section{Chapter 8}

\section{Conclusions}

\subsection{Summary and Conclusions}

In this thesis, we proposed an additional non-fading mode to an existing wireless fading network. We presented Shannon's channel capacity concepts, and provided some background on the AWGN and wireless fading channels. We also provided some background on diversity in wireless networks, with an emphasis on cooperative diversity.

For a wireless network consisting of $m$ transmitters and a destination $d(s)$, we presented modifications of two existing protocols for cooperative diversity; an $m$-phase repetition cooperation scheme and a two-phase distributed space-time scheme. Each of the existing protocols maintain full diversity of order $m$, with the space-time scheme outperforming the repetition-based scheme.

We introduced an additional non-fading mode into the network for each of these protocols. First, we examined the addition of the non-fading link between the message source and one of the relays in the repetition-based protocol. We presented a simulation of this configuration, and developed outage probability approximations for this configuration. We note that the system appears to achieve optimal outage when the source node directs enough power to the non-fading mode to ensure it achieves the target rate, and no more.

We then analyzed a non-fading link placed between a relay and the destination. Unfortunately, a closed form expression was not found. We presented our simulated results for the repetition relay-wire configuration. 
The outage probability for a space-time scheme with a non-fading mode between the source and relay was then studied. It was found, as in the repetition case, the system maintains full diversity and also seems to perform optimally at the point where enough power is dedicated to the non-fading mode to guarantee decoding. Unlike the repetition case, high-SNR approximations were developed for a non-fading mode placed between a relay and the destination. Again, this configuration was shown to achieve full-diversity. Also, in this configuration our results show power allocation need not be as precise as the previous configuration to achieve optimal outage performance.

In general, we can view the results of an additional non-fading mode as the ability to shape the $\lambda$ parameters describing the fading channel quality. For a system designer with the freedom to introduce a non-fading mode into the network, the fading channel can be effectively altered to the designer's specification. The non-fading mode allows for some customization of the fading channel's random nature.

\subsection{Future Work}

Our work did not consider non-fading modes placed in source-relay links in addition to relay-destination links, although it seems to be a straightforward extension of the work. Both the repetition and space-time schemes exclude the inter-relay links. It may be possible to develop a system where relays use the transmissions of other relays to their advantage.

While not mentioned in the paper, the maximum multiplexing gain possible is $1 / 2$, achieved by the space-time protocol. Existing cooperative protocols, such as that shown in [17], can achieve multiplexing gains closer to unity. It would be interesting to see if considering non-fading modes in such a network might impact performance.

A large-scale analysis of multimodal networks remains an important portion of remaining work. In a general wireless network, the question of where an additional non-fading mode would be best placed, as well as quantitative tools for analyzing the performance impact have yet to be developed. When designing large systems that must optimize across fading and non-fading modes these tools will be necessary.

In our model, the non-fading mode is used only for achieving the target rate of the 
system. The mode could also be used as a channel for automatic repeat requests, or ARQ. When the relay connected to the non-fading mode is unable to decode the transmission, the non-fading mode could be used as a feedback channel to request a re-transmission. An analysis of how achievable rates or other characteristics of the system are impacted would be a valuable addition. 


\section{References}

[1] G. Foschini and M. Gans, "On the Limits of wireless communications in a fading environment when using multiple antennas," Wireless Personal Commun, vol. 6, no. 3, pp. 311-335, 1998.

[2] CITA, "U.s. wireless quick facts," Mar. 2011.

[3] P. Gupta and P. R. Kumar, "The capacity of wireless networks," IEEE Trans. Inf. Theory, vol. 46, no. 2, pp. 388-404, Mar 2000.

[4] M. Franceschetti M. D. Migliore and P. Minero, "The capacity of wireless networks: information-theoretic and physical limits," IEEE Trans. Inf. Theory, vol. 55, no. 8, pp. 3413-24, Aug 2009.

[5] C.E. Shannon, "A mathematical theory of communication," Bell Systems Technical Journal, vol. 27, pp. 379-423, July 1948.

[6] H. Nyquist, "Thermal agitation of electric charge in conductors," Phys. Rev., vol. 32, no. 1, pp. 110-113, Jul 1928.

[7] T.M. Cover and J.A. Thomas, Elements of Information Theory, Wiley-Interscience, second edition, 2006.

[8] J.G. Proakis, Digital Communications, 3rd ed., New York, NY: McGraw-Hill, 1995.

[9] Andrea Goldsmith, Wireless Communications, Cambridge University Press, New York, NY, USA, 2005.

[10] G. Foschini, "Layered space-time architecture for wireless communication in a fading environment when using multi-element antennas.," Bell Labs. Tech. Journal, vol. 1, no. 2, pp. 41-59, Autumn 1996.

[11] L. Zheng, D. Tse, "Diversity and multiplexing: A fundamental tradeoff in multiple antenna channels," IEEE Transactions on Inform. Theory, vol. 49, pp. 1073-1096, May 2003.

[12] T. Cover and A.E. Gamal, "Capacity theorems for the relay channel," Information Theory, IEEE Transactions on, vol. 25, no. 5, pp. 572 - 584, Sept. 1979. 
[13] J. Winters, "On the capacity of radio communication systems with diversity in a rayleigh fading environment," Selected Areas in Communications, IEEE Journal on, vol. 5, no. 5, pp. $871-878$, June 1987.

[14] A. Sendonaris, E. Erkip, B. Aazang, "User cooperative diversity - Part I: System description," IEEE Trans. Communications, vol. 51, no. 1, pp. 1927-1938, Nov. 2003.

[15] A. Sendonaris, E. Erkip, B. Aazang, "User cooperative diversity - Part II: Implementation aspects and performance analysis," IEEE Trans. Communications, vol. 51, no. 1, pp. 1939-1948, Nov. 2003.

[16] N. Laneman and G. Wornell, "Distributed space time coded protocols for exploiting cooperative diversity in wireless networks," IEEE Tran. Info. Theory, vol. 49, no. 10, pp. 2415-2425, Oct. 2003.

[17] Yijia Fan, Chao Wang, J. Thompson, and H.V. Poor, "Recovering multiplexing loss through successive relaying using repetition coding," Wireless Communications, IEEE Transactions on, vol. 6, no. 12, pp. 4484 -4493, 2007. 


\section{A Additional Source-Relay Non-Fading Mode Approx- imation for Repetition}

We examine the effects of placing the additional mode between the source $s$ and one relay $r_{i}, \quad 1 \leq i \leq m-1$. We start with the definition of outage probability. Using the law of total probability, we partition the outage over all possible decoding sets, $\mathcal{D}(s)$,

$$
\operatorname{Pr}[\mathbf{I}<R]=\sum_{\mathcal{D}(s)} \operatorname{Pr}[\mathbf{I}<R \mid \mathcal{D}(s)] \operatorname{Pr}[\mathcal{D}(s)]
$$

(5.3) shows $I_{\text {rep }}$ conditioned on a decoding set. For the moment, we'll set aside the term $\operatorname{Pr}\left[I_{\text {rep }}<R \mid \mathcal{D}(s)\right]$ and focus on $\operatorname{Pr}[\mathcal{D}(s)]$. The decoding set is based on each relay $r_{i}$ decoding. If $s$ is connected to a non-fading mode in addition to communicating over the wireless channel it must divide its transmit power between the two modes. As mentioned previously, we use $P_{w}$ to denote the percentage of power dedicated to the non-fading mode. Then, $s$ provides transmit SNR $\left(1-P_{w}\right)$ SNR over the wireless mode.

Ignoring the relay connected to the non-fading mode for the moment, for a purely wireless relay $r_{i}$ to participate in the decoding set it must experience a minimum received SNR. Specifically, for a relay to decode messages sent at given rate $R$,

$$
\begin{aligned}
\frac{1}{m} \log \left(1+\left(1-P_{w}\right) \operatorname{SNR}\left|a_{s, r_{i}}\right|^{2}\right) & \geq R \\
\left|a_{s, r_{i}}\right|^{2} & \geq \frac{2^{m R}-1}{\left(1-P_{w}\right) \operatorname{SNR}} .
\end{aligned}
$$

$a_{s, r_{i}}$ is a Rayleigh random variable with parameter $\lambda_{s, r_{i}}$. Recall from Chapter 3 that squaring the magnitude of a Rayleigh random variable results in an exponential random variable. Thus, $\left|a_{s, r_{i}}\right|^{2}$ is an exponential random variable distributed with parameter $\lambda_{s, r_{i}}$. Using A2 ) and the CDF of an exponential random variable given by (3.5), the probability that relay $r_{i}$ participates in $\mathcal{D}(s)$, is

$$
\begin{aligned}
\operatorname{Pr}\left[r_{i} \in \mathcal{D}(s)\right] & =\operatorname{Pr}\left[\left|a_{s, r_{i}}\right|^{2}>\left(2^{m R}-1\right) /\left(\left(1-P_{w}\right) \text { SNR }\right)\right] \\
& =1-\left(1-\exp \left[-\lambda_{s, r_{i}} \frac{2^{m R}-1}{\left(1-P_{w}\right) \text { SNR }}\right]\right) \\
& =\exp \left[-\lambda_{s, r_{i}} \frac{2^{m R}-1}{\left(1-P_{w}\right) \operatorname{SNR}}\right]
\end{aligned}
$$


Conversely, the probability that $r_{i}$ is not able to decode is

$$
\begin{aligned}
\operatorname{Pr}\left[r_{i} \notin \mathcal{D}(s)\right] & =1-\operatorname{Pr}\left[r_{i} \in \mathcal{D}(s)\right] \\
& =1-\exp \left[-\lambda_{s, r_{i}} \frac{2^{m R}-1}{\left(1-P_{w}\right) \mathrm{SNR}}\right] .
\end{aligned}
$$

An instance of a decoding set $\mathcal{D}(s)$ consists of the nodes that are able to decode. Each relay experiences independent fading, therefore each relay determines it's ability to decode independently. Therefore, the probability of any specific decoding set $\mathcal{D}(s)$ is

$$
\operatorname{Pr}[\mathcal{D}(s)]=\prod_{r \in \mathcal{D}(s)} \exp \left[-\lambda_{s, r} \frac{2^{m R}-1}{\left(1-P_{w}\right) \operatorname{SNR}}\right] \times \prod_{r \notin \mathcal{D}(s)}\left(1-\exp \left[-\lambda_{s, r} \frac{2^{m R}-1}{\left(1-P_{w}\right) \operatorname{SNR}}\right]\right)
$$

We appeal to the Taylor series for a first-order approximation. Recall that for an exponential, the Taylor series expansion is $\exp (x)=1+x / 1 !+x^{2} / 2 !+x^{3} / 3 !+\ldots$ Assuming a first-order approximation of the exponential (A-5) becomes

$$
\operatorname{Pr}[\mathcal{D}(s)] \sim \prod_{r \in \mathcal{D}(s)}\left(1-\lambda_{s, r} \frac{2^{m R}-1}{\left(1-P_{w}\right) \operatorname{SNR}}\right) \times \prod_{r \notin \mathcal{D}(s)} \lambda_{s, r} \frac{2^{m R}-1}{\left(1-P_{w}\right) \operatorname{SNR}}
$$

At high-SNR one is the dominant term in the first product, and we assume the entire first product goes to unity. However, one is not the dominant term in the second product, thus we use it for the final approximation of $\operatorname{Pr}[\mathcal{D}(s)]$

$$
\operatorname{Pr}[\mathcal{D}(s)] \sim\left[\frac{2^{m R}-1}{\left(1-P_{w}\right) \operatorname{SNR}}\right]^{m-|\mathcal{D}(s)|-1} \times \prod_{r \notin \mathcal{D}(s)} \lambda_{s, r} .
$$

There are $m-|\mathcal{D}(s)|-1$ relays that don't decode, where $|\mathcal{D}(s)|$ is the cardinality of the set $\mathcal{D}(s)$, hence the exponent.

Up to this point, we have not considered the non-fading mode connected to $s$. and $\left(P_{w} B_{w l}\right) / B_{w l}$ SNR over the non-fading mode.

Consider placing the additional mode between $s$ and one of the relays, which we'll now call $r_{w}$. The relay $r_{w}$ will receive information over both non-fading and fading channels. As mentioned above, $r_{w}$ will experience an SNR $\left(P_{w} B_{w l}\right) / B_{w l}$ SNR over the fading mode. However, it will also receive an $\operatorname{SNR}\left(P_{w} B_{w l}\right) / B_{w l}$ SNR over the non-fading mode. The mutual information between this node and $s$ is now of a different form than (5.3),

$$
\begin{aligned}
\mathrm{I}_{\mathrm{s}, \mathrm{r}_{\mathrm{w}}}= & \frac{1}{m} \log \left(1+\left(1-P_{w}\right) \operatorname{SNR}\left|a_{s, r_{w}}\right|^{2}\right) \\
& +\frac{B_{w}}{m B_{w l}} \log \left(1+\frac{|h|^{2} P_{w} B_{w l}}{B_{w}} \operatorname{SNR}\right) .
\end{aligned}
$$


The information is a sum of the information available over the wireless channel (the first term) and the non-fading mode (the second term). Inspecting the second term, it is entirely deterministic. The probability that $r_{w}$ is included in the decoding set is

$$
\begin{aligned}
\operatorname{Pr}\left[r_{w} \in \mathcal{D}(s)\right]= & \operatorname{Pr}\left[\mathrm{I}_{\text {reps }, \mathrm{r}_{\mathrm{w}}}>R\right] \\
= & \operatorname{Pr}\left[\frac{1}{m} \log \left(1+\left(1-P_{w}\right) \operatorname{SNR}\left|a_{s, r_{w}}\right|^{2}\right)\right. \\
& \left.+\frac{B_{w}}{m B_{w l}} \log \left(1+\frac{|h|^{2} P_{w} B_{w l}}{B_{w}} \mathrm{SNR}\right)>R\right] \\
= & \operatorname{Pr}\left[\frac{1}{m} \log \left(1+\left(1-P_{w}\right) \operatorname{SNR}\left|a_{s, r_{w}}\right|^{2}\right)\right. \\
& \left.>R-\frac{B_{w}}{m B_{w l}} \log \left(1+\frac{|h|^{2} P_{w} B_{w l}}{B_{w}} \mathrm{SNR}\right)\right]
\end{aligned}
$$

After shifting the deterministic term $\frac{B_{w}}{m B_{w l}} \log \left(1+\frac{|h|^{2} P_{w} B_{w l}}{B_{w}}\right.$ SNR $)$ to the right hand side of the inequality, (A-9) looks very similar to A-3). Essentially, the relay experiences a rate-reduction required to decode. Performing the substitution $\hat{R}=R-\frac{B_{w}}{m B_{w l}} \log (1+$ $\frac{|h|^{2} P_{w} B_{w l}}{B_{w}}$ SNR $)$, the equation becomes

$$
\begin{aligned}
\operatorname{Pr}\left[r_{w} \in \mathcal{D}(s)\right] & =\operatorname{Pr}\left[\frac{1}{m} \log \left(1+\left(1-P_{w}\right) \operatorname{SNR}\left|a_{s, r_{w}}\right|^{2}\right)>\hat{R}\right] \\
& =\operatorname{Pr}\left[\left|a_{s, r_{w}}\right|^{2}>\frac{2^{m \hat{R}}-1}{\left(1-P_{w}\right) \operatorname{SNR}}\right] .
\end{aligned}
$$

Note that $\mathrm{A}-10$ is now of the form of $\mathrm{A}-3$. This means $\operatorname{Pr}\left[r_{w} \in \mathcal{D}(s)\right]$ is also an exponential random variable. We can then write

$$
\operatorname{Pr}\left[r_{w} \in \mathcal{D}(s)\right]=\exp \left[-\lambda_{s, r_{i}} \frac{2^{m R}-1}{\left(1-P_{w}\right) \operatorname{SNR}}\right]
$$

and $\operatorname{Pr}\left[r_{w} \notin \mathcal{D}(s)\right]$ is

$$
\operatorname{Pr}\left[r_{w} \notin \mathcal{D}(s)\right]=1-\left(\exp \left[-\lambda_{s, r_{i}} \frac{2^{m R}-1}{\left(1-P_{w}\right) \operatorname{SNR}}\right]\right) .
$$

We now move to the other term in $\mathrm{A}-1), \operatorname{Pr}[\mathrm{I}<R \mid \mathcal{D}(s)]$. Fortunately, this term is unaffected by placing an additional non-fading mode between $s$ and $r_{w}$. The additional mode affects only $\operatorname{Pr}[\mathcal{D}(s)]$. Assuming $\mathcal{D}(s)$ has already been determined, we can use the approximation developer in [16] without modification. $\operatorname{Pr}\left[\mathrm{I}_{\text {rep }}<R \mid \mathcal{D}(s)\right]$ can be approximated 
as

$$
\operatorname{Pr}\left[\mathrm{I}_{\text {rep }}<R \mid \mathcal{D}(s)\right] \sim\left[\frac{2^{m R}-1}{\mathrm{SNR}}\right]^{|\mathcal{D}(s)|+1} \times \lambda_{s, d(s)} \prod_{r \in \mathcal{D}(s)} \lambda_{r, d(s)} \times \frac{1}{(|\mathcal{D}(s)|+1) !} .
$$

Returning now to $\mathrm{A}-1$, we further subdivide the partition over all decoding sets to two distinct partitions; decoding sets that include $r_{w}$ and those that don't.

$$
\begin{aligned}
\operatorname{Pr}\left[\left.\right|_{\text {rep }}<R\right] & =\sum_{\substack{\mathcal{D}(s): \\
r_{w} \in \mathcal{D}(s)}} \operatorname{Pr}[1<R \mid \mathcal{D}(s)] \operatorname{Pr}[\mathcal{D}(s)] \\
& +\sum_{\substack{\mathcal{D}(s): \\
r_{w} \notin \mathcal{D}(s)}} \operatorname{Pr}[1<R \mid \mathcal{D}(s)] \operatorname{Pr}[\mathcal{D}(s)]
\end{aligned}
$$

(A-14 is equivalent to $\mathrm{A}-1$. Using $\mathrm{A}-11$ and $\mathrm{A}-12$ we can write

$$
\begin{aligned}
\operatorname{Pr}\left[\mathcal{D}(s): r_{w} \in \mathcal{D}(s)\right] \sim & \exp \left[-\lambda_{s, r_{i}} \frac{2^{m R}-1}{\left(1-P_{w}\right) \mathrm{SNR}}\right] \\
& \times\left[\frac{2^{m R}-1}{\left(1-P_{w}\right) \operatorname{SNR}}\right]^{m-|\mathcal{D}(s)|-1} \times \prod_{r \notin \mathcal{D}(s)} \lambda_{s, r},
\end{aligned}
$$

and

$$
\begin{aligned}
\operatorname{Pr}\left[\mathcal{D}(s): r_{w} \notin \mathcal{D}(s)\right] \sim & \left\{1-\left(\exp \left[-\lambda_{s, r_{i}} \frac{2^{m R}-1}{\left(1-P_{w}\right) \operatorname{SNR}}\right]\right)\right\} \\
& \times\left[\frac{2^{m R}-1}{\left(1-P_{w}\right) \operatorname{SNR}}\right]^{m-|\mathcal{D}(s)|-1} \times \prod_{r \notin \mathcal{D}(s)} \lambda_{s, r} .
\end{aligned}
$$

Leveraging the Taylor series expansion the first terms in each equation can be simplified to

$$
\operatorname{Pr}\left[\mathcal{D}(s): r_{w} \in \mathcal{D}(s)\right] \sim\left[\frac{2^{m R}-1}{\left(1-P_{w}\right) \operatorname{SNR}}\right]^{m-|\mathcal{D}(s)|-1} \times \prod_{r \notin \mathcal{D}(s)} \lambda_{s, r},
$$

and

$$
\begin{aligned}
\operatorname{Pr}\left[\mathcal{D}(s): r_{w} \notin \mathcal{D}(s)\right] \sim & \lambda_{s, r_{w}} \times\left[\frac{2^{m \hat{R}}-1}{\left(1-P_{w}\right) \operatorname{SNR}}\right] \times\left[\frac{2^{m R}-1}{\left(1-P_{w}\right) \operatorname{SNR}}\right]^{m-|\mathcal{D}(s)|-2} \\
& \times \prod_{\substack{r \notin \mathcal{D}(s) \\
r \neq r_{w}}} \lambda_{s, r}
\end{aligned}
$$

respectively. 
Substituting equations A-15 and A-16 back into A-14, we achieve the following result

$$
\begin{aligned}
& \operatorname{Pr}\left[\mathrm{I}_{\text {rep }}<R\right] \sim\left[\frac{2^{m R}-1}{\mathrm{SNR}}\right]^{m} \times \lambda_{s, d(s)} \times\left\{\sum_{\substack{\mathcal{D}(s): \\
r_{w} \in \mathcal{D}(s)}}\left[\frac{1}{1-P_{w}}\right]^{m-|\mathcal{D}(s)|-1}\right. \\
& \times \prod_{r \in \mathcal{D}(s)} \lambda_{r, d(s)} \times \prod_{r \notin \mathcal{D}(s)} \lambda_{s, r} \times \frac{1}{(|\mathcal{D}(s)|+1) !} \\
& +\sum_{\substack{\mathcal{D}(s): \\
r_{w} \notin \mathcal{D}(s)}}\left[\frac{2^{m \hat{R}}-1}{2^{m R}-1}\right] \lambda_{s, r_{w}} \times\left[\frac{1}{1-P_{w}}\right]^{m-|\mathcal{D}(s)|-1} \\
& \left.\times \prod_{r \in \mathcal{D}(s)} \lambda_{r, d(s)} \times \prod_{\substack{r \notin \mathcal{D}(s) \\
r \neq r_{w}}} \lambda_{s, r} \times \frac{1}{(|\mathcal{D}(s)|+1) !}\right\} .
\end{aligned}
$$

Letting $\bar{\lambda}_{s, r}=\lambda_{s, r}$ for all $r \neq r_{w}$, and $\bar{\lambda}_{s, r_{w}}=\left(2^{m \hat{R}}-1\right) /\left(2^{m R}-1\right) \lambda_{s, r_{w}}$, we arrive at (5.8).

\section{B Additional Source-Relay Non-Fading Mode for Space- Time}

With two modes of communication available, $s$ must divide its finite power between the fading and non-fading mode. $P_{w}$ will denote the percentage of power dedicated to the non-fading mode, with $P_{w} \in[0,1]$. Since other relays are attempting to decode the transmission from $s$, as $P_{w}$ increases the SNR for transmission over the wireless fading link to all other relays will decrease. Relays not connected to the non-fading mode will experience an instantaneous mutual information

$$
\mathrm{I}_{\mathrm{s}, \mathrm{r}}=\frac{1}{2} \log \left(1+\left(1-P_{w}\right) \frac{2}{m} \operatorname{SNR}\left|a_{s, r}\right|^{2}\right), r \neq r_{w} .
$$

For these relays not connected to the additional non-fading mode to decode, $\mathrm{I}_{\mathrm{s}, \mathrm{r}}$ must be greater than the target rate $R$. The probability that these relays participate in the decoding set $\mathcal{D}(s)$ is

$$
\begin{aligned}
\operatorname{Pr}[r \in \mathcal{D}(s)] & =\operatorname{Pr}\left[\mathrm{I}_{\mathrm{s}, \mathrm{r}}>R\right] \\
& =\operatorname{Pr}\left[\frac{1}{2} \log \left(1+\left(1-P_{w}\right) \frac{2}{m} \operatorname{SNR}\left|a_{s, r}\right|^{2}\right)>R\right] \\
& =\operatorname{Pr}\left[\left|a_{s, r}\right|^{2}>\left(\frac{2^{2 R}-1}{\left(1-P_{w}\right) 2 \mathrm{SNR} / m}\right)\right], r \neq r_{w} .
\end{aligned}
$$


Note that $(\mathrm{B}-21)$ is the $\mathrm{CDF}$ of an exponential random variable.

The mutual information for $r_{w}$ will be from both the wireless fading mode and the nonfading mode,

$$
\mathrm{I}_{\mathrm{s}, \mathrm{r}_{\mathrm{w}}}=\frac{1}{2} \log \left(1+\left(1-P_{w}\right) \frac{2}{m} \operatorname{SNR}\left|a_{s, r_{w}}\right|^{2}\right)+\frac{B_{w}}{2 B_{w l}} \log \left(1+\frac{P_{w} B_{w l}}{B_{w}} \frac{2}{m} \operatorname{SNR}|h|^{2}\right) .
$$

For $r_{w}$ to participate in the decoding set $\mathcal{D}(s)$, it must achieve the target rate $R$. The probability that $r_{w}$ participates in $\mathcal{D}(s)$ is

$$
\begin{aligned}
\operatorname{Pr}\left[r_{w} \in \mathcal{D}(s)\right]= & \operatorname{Pr}\left[\mathrm{I}_{\mathrm{s}, \mathrm{r}_{w}}>R\right] \\
= & \operatorname{Pr}\left[\frac{1}{2} \log \left(1+\left(1-P_{w}\right) \frac{2}{m} \mathrm{SNR}\left|a_{s, r_{w}}\right|^{2}\right)\right. \\
& \left.+\frac{B_{w}}{2 B_{w l}} \log \left(1+\frac{P_{w} B_{w l}}{B_{w}} \frac{2}{m} \mathrm{SNR}|h|^{2}\right)>R\right] \\
= & \operatorname{Pr}\left[\frac{1}{2} \log \left(1+\left(1-P_{w}\right) \frac{2}{m} \mathrm{SNR}\left|a_{s, r_{w}}\right|^{2}\right)>R\right. \\
& \left.-\frac{B_{w}}{2 B_{w l}} \log \left(1+\frac{P_{w} B_{w l}}{B_{w}} \frac{2}{m} \mathrm{SNR}|h|^{2}\right)\right] .
\end{aligned}
$$

As before, the term moved to the right-hand-side of the equation is entirely deterministic. Letting $\hat{R}=R-\frac{B_{w}}{2 B_{w l}} \log \left(1+\frac{P_{w} B_{w l}}{B_{w}} \frac{2}{m} \mathrm{SNR}|h|^{2}\right)$, and substituting $\hat{R}$ into B-23 yields

$$
\begin{aligned}
\operatorname{Pr}\left[r_{w} \in \mathcal{D}(s)\right] & =\operatorname{Pr}\left[\frac{1}{2} \log \left(1+\left(1-P_{w}\right) \frac{2}{m} \operatorname{SNR}\left|a_{s, r_{w}}\right|^{2}\right)>\hat{R}\right] \\
& =\operatorname{Pr}\left[\left|a_{s, r_{w}}\right|^{2}>\left(\frac{2^{2 \hat{R}}-1}{\left(1-P_{w}\right) 2 \mathrm{SNR} / m}\right)\right] .
\end{aligned}
$$

Again, as in the previous chapter, $(\bar{B}-24)$ is the CDF of an exponential random variable, much like (B-21), but with reduced rate $\hat{R}$. With the probabilities for $r_{w}$ and the other relays $r$ for participating in the decoding set, we proceed with defining the outage probability.

Again, we use the law of total probability to define outage probability of the network as shown in $\mathrm{A}-1$. Since each relay in the network experiences independent fading, the probability of a specific decoding set $\mathcal{D}(s)$ is

$$
\operatorname{Pr}[\mathcal{D}(s)]=\prod_{r \in \mathcal{D}(s)} \operatorname{Pr}[r \in \mathcal{D}(s)] \times \prod_{r \notin \mathcal{D}(s)} \operatorname{Pr}[r \notin \mathcal{D}(s)]
$$

We again partition the summation in $(\mathrm{A}-1)$ in two; decoding sets that include $r_{w}$ and those that don't as shown in $\mathrm{A}-14$. 
We analyze the probability of the first partition, $\operatorname{Pr}\left[\mathcal{D}(s): r_{w} \in \mathcal{D}(s)\right]$,

$$
\begin{aligned}
\operatorname{Pr}\left[\mathcal{D}(s): r_{w} \in \mathcal{D}(s)\right]= & \operatorname{Pr}\left[\left|a_{s, r_{w}}\right|^{2}>\left(\frac{2^{2 \hat{R}}-1}{\left(1-P_{w}\right) 2 \mathrm{SNR} / m}\right)\right] \\
& \times \prod_{\substack{r \in \mathcal{D}(s): \\
r \neq r_{w}}} \operatorname{Pr}\left[\left|a_{s, r}\right|^{2}>\left(\frac{2^{2 R}-1}{\left(1-P_{w}\right) 2 \mathrm{SNR} / m}\right)\right] \\
& \times \prod_{r \notin \mathcal{D}(s)}\left(1-\operatorname{Pr}\left[\left|a_{s, r}\right|^{2}>\left(\frac{2^{2 R}-1}{\left(1-P_{w}\right) 2 \mathrm{SNR} / m}\right)\right]\right) .
\end{aligned}
$$

And similarly we can write $\operatorname{Pr}\left[\mathcal{D}(s): r_{w} \notin \mathcal{D}(s)\right]$

$$
\begin{aligned}
\operatorname{Pr}\left[\mathcal{D}(s): r_{w} \notin \mathcal{D}(s)\right]= & \left(1-\operatorname{Pr}\left[\left|a_{s, r_{w}}\right|^{2}>\left(\frac{2^{2 \hat{R}}-1}{\left(1-P_{w}\right) 2 \mathrm{SNR} / m}\right)\right]\right) \\
& \times \prod_{r \in \mathcal{D}(s)} \operatorname{Pr}\left[\left|a_{s, r}\right|^{2}>\left(\frac{2^{2 R}-1}{\left(1-P_{w}\right) 2 \mathrm{SNR} / m}\right)\right] \\
& \times \prod_{\substack{r \notin \mathcal{D}(s) \\
r \neq r_{w}}}\left(1-\operatorname{Pr}\left[\left|a_{s, r}\right|^{2}>\left(\frac{2^{2 R}-1}{\left(1-P_{w}\right) 2 \mathrm{SNR} / m}\right)\right]\right) .
\end{aligned}
$$

Each of these probabilities represent exponential random variables, or the complement of an exponential random variable, and we use the Taylor series for a first-order approximation. We arrive at the following two equations,

$$
\operatorname{Pr}\left[\mathcal{D}(s): r_{w} \in \mathcal{D}(s)\right] \sim\left[\frac{2^{2 R}-1}{\left(1-P_{w}\right) 2 \mathrm{SNR} / m}\right]^{m-|\mathcal{D}(s)|-1} \times \prod_{r \notin \mathcal{D}(s)} \lambda_{s, r}
$$

and

$$
\begin{aligned}
\operatorname{Pr}\left[\mathcal{D}(s): r_{w} \notin \mathcal{D}(s)\right] \sim & {\left[\frac{2^{2 R}-1}{\left(1-P_{w}\right) 2 \mathrm{SNR} / m}\right]^{m-|\mathcal{D}(s)|-2} \times\left[\frac{2^{2 \hat{R}}-1}{\left(1-P_{w}\right) 2 \mathrm{SNR} / m}\right] } \\
& \times \lambda_{s, r_{w}} \times \prod_{\substack{r \notin \mathcal{D}(s) \\
r \neq r_{w}}} \lambda_{s, r} \\
\sim & {\left[\frac{2^{2 R}-1}{\left(1-P_{w}\right) 2 \mathrm{SNR} / m}\right]^{m-|\mathcal{D}(s)|-1} \times\left[\frac{2^{2 \hat{R}}-1}{2^{2 R}-1}\right] \lambda_{s, r_{w}} } \\
& \times \prod_{\substack{r \notin \mathcal{D}(s) \\
r \neq r_{w}}} \lambda_{s, r}
\end{aligned}
$$

Once again, as was the case in chapter 5, when $r_{w}$ is included in the decoding set the approximation of $\operatorname{Pr}[\mathcal{D}(s)]$ is the same. Also, if we consider $\lambda_{s, r_{w}}\left(2^{2 \hat{R}}-1\right) /\left(2^{2 R}-1\right)$ a 
weighted version of the channel quality, in essence the expression does not change. $\operatorname{Pr}[\mathcal{D}(s)]$ still does not change, and is still the product of all lambda parameters of relays excluded from the decoding set.

For the two-phase network, an additional non-fading mode between $s$ and a relay $r$ has no impact on the second stage of communication. The non-fading mode will only impact $\operatorname{Pr}[\mathcal{D}(s)]$. The second stage of communication, where all nodes in $\mathcal{D}(s)$ transmit simultaneously, is conditioned on the decoding set. With this in mind, we can use $\operatorname{Pr}[I<R \mid \mathcal{D}(s)]$ for DSTC developed in [16] without additional modification.

Substituting equations B-28 and B-29 back into (A-14) yields

$$
\begin{aligned}
\operatorname{Pr}[\mathrm{I}<R] \sim & \sum_{\substack{\mathcal{D}(s): \\
r_{w} \in \mathcal{D}(s)}} \operatorname{Pr}[\mathrm{I}<R \mid \mathcal{D}(s)]\left[\frac{2^{2 R}-1}{\left(1-P_{w}\right) 2 \mathrm{SNR} / m}\right]^{m-|\mathcal{D}(s)|-1} \times \prod_{r \notin \mathcal{D}(s)} \lambda_{s, r} \\
+ & \sum_{\substack{\mathcal{D}(s): \\
r_{w} \notin \mathcal{D}(s)}} \operatorname{Pr}[\mathrm{I}<R \mid \mathcal{D}(s)]\left[\frac{2^{2 R}-1}{\left(1-P_{w}\right) 2 \mathrm{SNR} / m}\right]^{m-|\mathcal{D}(s)|-1} \\
& \times\left[\frac{2^{2 \hat{R}}-1}{2^{2 R}-1}\right] \lambda_{s, r_{w}} \times \prod_{\substack{r \notin \mathcal{D}(s) \\
r \neq r_{w}}} \lambda_{s, r} .
\end{aligned}
$$


Finally, substituting $\operatorname{Pr}[I<R \mid \mathcal{D}(s)] \sim\left[\left(2^{2 R}-1\right) /(2 \mathrm{SNR} / m)\right]^{|\mathcal{D}(s)|+1} \times \lambda_{s, d(s)} \times \prod_{r \in \mathcal{D}(s)} \lambda_{r, d(s)} \times$ $A_{|\mathcal{D}(s)|}\left(2^{2 R}-1\right)$ from [16], equation (19) yields

$$
\begin{aligned}
\operatorname{Pr}[\mathrm{I}<R] \sim & {\left[\frac{2^{2 R}-1}{2 \mathrm{SNR} / m}\right]^{m} \times \lambda_{s, d(s)} } \\
& \times\left\{\sum_{\substack{\mathcal{D}(s): \\
r_{w} \in \mathcal{D}(s)}}\left[\frac{1}{1-P_{w}}\right]^{m-|\mathcal{D}(s)|-1} \times \prod_{r \in \mathcal{D}(s)} \lambda_{r, d(s)} \times \prod_{r \notin \mathcal{D}(s)} \lambda_{s, r} \times A_{|\mathcal{D}(s)|}\left(2^{2 R}-1\right)\right. \\
& +\sum_{\substack{\mathcal{D}(s): \\
r_{w} \in \mathcal{D}(s)}}\left[\frac{1}{1-P_{w}}\right]^{m-|\mathcal{D}(s)|-1} \times \prod_{r \in \mathcal{D}(s)} \lambda_{r, d(s)} \times \prod_{\substack{r \notin \mathcal{D}(s): \\
r \neq r_{w}}} \lambda_{s, r} \\
& \left.\times\left[\frac{2^{2 \hat{R}}-1}{2^{2 R}-1}\right] \lambda_{s, r_{w}} \times A_{|\mathcal{D}(s)|}\left(2^{2 R}-1\right)\right\} .
\end{aligned}
$$

Letting $\bar{\lambda}_{s, r}=\lambda_{s, r}$ for all $r \neq r_{w}$. Let $\bar{\lambda}_{s, r_{w}}=\left(2^{2 \hat{R}}-1\right) /\left(2^{2 R}-1\right) \lambda_{s, r_{w}}$, we arrive at 7.13 .

\section{Additional Relay-Destination Non-Fading Mode for Space-Time}

We begin by examining the mutual information at the destination $d(s)$ after the secondphase of communication. In [16], it was shown the mutual information at $d(s)$ in absence of a non-fading mode is

$$
\mathbf{I}=\frac{1}{2} \log \left(1+\frac{2}{m} \operatorname{SNR}\left|a_{s, d(s)}\right|^{2}\right)+\frac{1}{2} \log \left(1+\frac{2}{m} \operatorname{SNR} \sum_{r \in \mathcal{D}(s)}\left|a_{r, d(s)}\right|^{2}\right) .
$$

With a non-fading mode present, the expression becomes

$$
\begin{aligned}
\mathrm{I}= & \frac{1}{2} \log \left(1+\frac{2}{m} \operatorname{SNR}\left|a_{s, d(s)}\right|^{2}\right)+\frac{1}{2} \log \left(1+\frac{2}{m} \operatorname{SNR}\left[\left(1-P_{w}\right)\left|a_{r_{w}, d(s)}\right|^{2}+\sum_{\substack{r \in \mathcal{D}(s): \\
r \neq r_{w}}}\left|a_{r, d(s)}\right|^{2}\right]\right) \\
& +\frac{B_{w}}{2 B_{w l}} \log \left(1+\frac{2}{m B_{w} / B_{w l}} \operatorname{SNR}\right) .
\end{aligned}
$$

We again assume $r_{w}$ transmits in only the second-phase of communication across both modes, if and only if it decodes in the first-phase. Also, the non-fading mode has bandwidth $B_{w}$, so the term $B_{w} /\left(2 B_{w l}\right)$ is used to normalize the spectral efficiency of the last term with respect to bandwidth and degrees-of-freedom. 
We consider two cases for $\operatorname{Pr}[I<R \mid \mathcal{D}(s)], r_{w} \in \mathcal{D}(s)$ and $r_{w} \notin \mathcal{D}(s)$. First, when $r_{w}$ successfully decodes the information at $d(s)$ is calculated as shown in (C-33). The probability of outage in this case becomes

$$
\begin{aligned}
\operatorname{Pr}\left[I<R \mid \mathcal{D}(s): r_{w} \in \mathcal{D}(s)\right]=\operatorname{Pr} & {\left[\frac{1}{2} \log \left(1+\frac{2}{m} \operatorname{SNR}\left|a_{s, d(s)}\right|^{2}\right)\right.} \\
& +\frac{1}{2} \log \left(1+\frac{2}{m} \operatorname{SNR}\left[\left(1-P_{w}\right)\left|a_{r_{w}, d(s)}\right|^{2}+\sum_{\substack{r \in \mathcal{D}(s): \\
r \neq r_{w}}}\left|a_{r, d(s)}\right|^{2}\right]\right) \\
& \left.+\frac{B_{w}}{2 B_{w l}} \log \left(1+\frac{2}{m B_{w} / B_{w l}} \operatorname{SNR}\right)<R\right] . \quad(\mathrm{C}-34)
\end{aligned}
$$

Shifting the deterministic term to the right-hand side of the inequality, we arrive at the following,

$$
\begin{aligned}
\operatorname{Pr}\left[I<R \mid \mathcal{D}(s): r_{w} \in \mathcal{D}(s)\right]=\operatorname{Pr} & {\left[\frac{1}{2} \log \left(1+\frac{2}{m} \operatorname{SNR}\left|a_{s, d(s)}\right|^{2}\right)\right.} \\
& +\frac{1}{2} \log \left(1+\frac{2}{m} \operatorname{SNR}\left[\left(1-P_{w}\right)\left|a_{r_{w}, d(s)}\right|^{2}+\sum_{\substack{r \in \mathcal{D}(s): \\
r \neq r_{w}}}\left|a_{r, d(s)}\right|^{2}\right]\right) \\
& \left.<R-\frac{B_{w}}{2 B_{w l}} \log \left(1+\frac{2}{m B_{w} / B_{w l}} \operatorname{SNR}\right)\right] .
\end{aligned}
$$

We denote this reduced-rate on the right-hand-side of the inequality as $\hat{R}=R-$ $\frac{B_{w}}{2 B_{w l}} \log \left(1+(2) /\left(m B_{w} / B_{w l}\right)\right.$ SNR $)$. We can now rewrite the previous equation as

$$
\begin{aligned}
\operatorname{Pr}\left[I<R \mid \mathcal{D}(s): r_{w} \in \mathcal{D}(s)\right]=\operatorname{Pr}[ & \frac{1}{2} \log \left(1+\frac{2}{m} \operatorname{SNR}\left|a_{s, d(s)}\right|^{2}\right) \\
& +\frac{1}{2} \log \left(1+\frac{2}{m} \operatorname{SNR}\left[\left(1-P_{w}\right)\left|a_{r_{w}, d(s)}\right|^{2}\right.\right. \\
& \left.\left.\left.+\sum_{\substack{r \in \mathcal{D}(s): \\
r \neq r_{w}}}\left|a_{r, d(s)}\right|^{2}\right]\right)<\hat{R}\right] \\
=\operatorname{Pr}\left[\left|a_{s, d(s)}\right|^{2}+\left(\left(1-P_{w}\right)\left|a_{r_{w}, d(s)}\right|^{2}+\sum_{\substack{r \in \mathcal{D}(s): \\
r \neq r_{w}}}\left|a_{r, d(s)}\right|^{2}\right)\right. & \\
& +\frac{2}{m} \operatorname{SNR}\left|a_{s, d(s)}\right|^{2}\left(\left(1-P_{w}\right)\left|a_{r_{w}, d(s)}\right|^{2}\right. \\
& \left.\left.+\sum_{\substack{r \in \mathcal{D}(s): \\
r \neq r_{w}}}\left|a_{r, d(s)}\right|^{2}\right)<\frac{2^{2 \hat{R}}-1}{2 \operatorname{SNR} / m}\right]
\end{aligned}
$$


Note that if $\left|a_{r_{w}, d(s)}\right|^{2}$ is an exponential random variable distributed with parameter $\lambda_{r_{w}, d(s)}$, then $\left(1-P_{w}\right)\left|a_{r_{w}, d(s)}\right|^{2}$ is also an exponential random variable distributed with parameter $\lambda_{r_{w}, d(s)} /\left(1-P_{w}\right)$. With that in mind, (C-36) is of the same form as [16], (50). Therefore, we can exploit [16], (19)

$$
\begin{aligned}
\operatorname{Pr}\left[I<R \mid \mathcal{D}(s): r_{w} \in \mathcal{D}(s)\right] \sim & {\left[\frac{2^{2 \hat{R}}-1}{2 \operatorname{SNR} / m}\right]^{|\mathcal{D}(s)|+1} \times \lambda_{s, d(s)} \times \frac{\lambda_{r_{w}, d(s)}}{\left(1-P_{w}\right)} } \\
& \times \prod_{\substack{r \in \mathcal{D}(s): \\
r \neq r_{w}}} \lambda_{r, d(s)} \times A_{|\mathcal{D}(s)|}\left(2^{2 \hat{R}}-1\right) .
\end{aligned}
$$

When $r_{w} \notin \mathcal{D}(s), \operatorname{Pr}[I<R \mid \mathcal{D}(s)]$ is the same as in [16], (19) without additional modification. We once again appeal to partitions of the outage probability, $\mathrm{A}-14$. Plugging in $\mathrm{C}-36$ the outage probability becomes

$$
\begin{aligned}
\operatorname{Pr}[\mid<R] \sim & \sum_{\substack{\mathcal{D}(s): \\
r_{w} \in \mathcal{D}(s)}}\left[\frac{2^{2 \hat{R}}-1}{2 \mathrm{SNR} / m}\right]^{|\mathcal{D}(s)|+1} \times \lambda_{s, d(s)} \times \frac{\lambda_{r_{w}, d(s)}}{\left(1-P_{w}\right)} \\
& \times \prod_{\substack{r \in \mathcal{D}(s): \\
r \neq r_{w}}} \lambda_{r, d(s)} \times A_{|\mathcal{D}(s)|}\left(2^{2 \hat{R}}-1\right) \times \operatorname{Pr}[\mathcal{D}(s)] \\
+ & \sum_{\substack{\mathcal{D}(s): \\
r_{w} \notin \mathcal{D}(s)}}\left[\frac{2^{2 R}-1}{2 \mathrm{SNR} / m}\right]^{|\mathcal{D}(s)|+1} \times \lambda_{s, d(s)} \\
& \times \prod_{\substack{r \in \mathcal{D}(s) \\
\lambda_{r, d(s)}}} \lambda_{|\mathcal{D}(s)|}\left(2^{2 R}-1\right) \times \operatorname{Pr}[\mathcal{D}(s)]
\end{aligned}
$$

Now, inserting $\operatorname{Pr}[\mathcal{D}(s)] \sim\left[\frac{2^{2 R_{-}}}{2 \mathrm{SNR} / m}\right]^{m-|\mathcal{D}(s)|-1} \times \prod_{r \notin \mathcal{D}(s)} \lambda_{s, r}$ from [16], (21) we have

$$
\begin{aligned}
\operatorname{Pr}[\mathbf{I}<R] \sim & {\left[\frac{2^{2 R}-1}{2 \mathrm{SNR} / m}\right]^{m} \times \lambda_{s, d(s)} } \\
\times & \left\{\sum_{\substack{\mathcal{D}(s): \\
r_{w} \in \mathcal{D}(s)}}\left[\frac{2^{2 \hat{R}}-1}{2^{2 R}-1}\right]^{|\mathcal{D}(s)|+1} \times \frac{\lambda_{r_{w}, d(s)}}{\left(1-P_{w}\right)}\right. \\
& \times \prod_{\substack{r \in \mathcal{D}(s): \\
r \neq r_{w}}} \lambda_{r, d(s)} \times \prod_{r \notin \mathcal{D}(s)} \lambda_{s, r} \times A_{|\mathcal{D}(s)|}\left(2^{2 \hat{R}}-1\right) \\
& +\sum_{\substack{\mathcal{D}(s): \\
r_{w} \notin \mathcal{D}(s)}} \prod_{r \notin \mathcal{D}(s)} \lambda_{s, r} \times \prod_{r \in \mathcal{D}(s)} \lambda_{r, d(s)} \\
& \left.\times A_{|\mathcal{D}(s)|}\left(2^{2 R}-1\right)\right\} .
\end{aligned}
$$

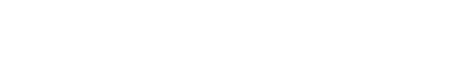
Virginia University Libraries, ou=Acquisitions Department,
email=John.Hagen@mail.wvued u, c=US
Date: 2011.05 .04 16:40:01 -04'00' 